\title{
Evidence for non-hadronic interactions of charm degrees of freedom in heavy-ion collisions at relativistic energies
}

\author{
O. Linnyk, ${ }^{a, *}$ E. L. Bratkovskaya, ${ }^{a}$ W. Cassing, ${ }^{b}$ \\ a Frankfurt Institute for Advanced Studies,60438 Frankfurt am Main,Germany \\ b Institut für Theoretische Physik, Universität Giessen,35392 Giessen, Germany
}

\begin{abstract}
Within the Hadron-String Dynamics (HSD) transport approach we study the suppression pattern of charmonia at RHIC with respect to centrality and rapidity employing various model concepts such as variants of the 'comover absorption' model or the 'charmonium melting' scenario. We find that especially the ratio of the forward to mid-rapidity nuclear modification factors of $J / \Psi\left(R_{A A}^{f o r w a r d}(J / \Psi) / R_{A A}^{\text {mid }}(J / \Psi)\right)$ cannot be explained by the interactions with 'formed' comoving mesons or by the 'color screening mechanism' alone. Only when incorporating interactions of the $c$ or $\bar{c}$ quark with a pre-hadronic medium satisfactory results are obtained. A detailed comparison to the PHENIX data demonstrates that non-hadronic interactions are mandatory to describe the narrowing of the $J / \Psi$ rapidity distribution from $p p$ to central $\mathrm{Au}+\mathrm{Au}$ collisions. The $\Psi^{\prime}$ to $J / \Psi$ ratio is found to be crucial in disentangling the different charmonium absorption scenarios especially in the RHIC energy range. Furthermore, a comparison of the transport calculations to the statistical model of Gorenstein and Gazdzicki as well as the statistical hadronization model of Andronic et al. shows differences in the energy dependence as well as centrality dependence of the $J / \Psi$ to pion ratio which may be exploited experimentally to disentangle different concepts. We find additionally that the collective flow of charm in the HSD transport appears compatible with the data at SPS energies but substantially underestimates the data at top RHIC energies such that the large elliptic flow $v_{2}$ of charm seen experimentally has to be attributed to early interactions of non-hadronic degrees of freedom.
\end{abstract}

Key words: Relativistic heavy-ion collisions, Meson production, Quark-gluon plasma, Charmed mesons, Charmed quarks

PACS 25.75.-q, 13.60.Le, 12.38.Mh, 14.40.Lb, 14.65.Dw

\footnotetext{
* corresponding author

Email address: linnyk@fias.uni-frankfurt.de (O. Linnyk,).
} 


\section{Introduction}

An investigation of the formation and suppression dynamics of $J / \Psi, \chi_{c}$ and $\Psi^{\prime}$ mesons opens the possibility to address fundamental questions about the properties of the state of matter at high temperature and density. Up to date, a simultaneous description of the seemingly energy-independent suppression of $J / \Psi$ together with its narrow rapidity distribution and a strong elliptic flow $v_{2}$ of charmed hadrons - as found at the Relativistic-Heavy-Ion-Collider (RHIC) - has presented a challenge to microscopic theories. The large discrepancies of present studies are striking in view of the success of the hadron-string transport theories in describing charmonium data at SPS energies. This has lead to the conjecture that the sizeable difference between the measured yields and transport predictions is due to a neglect of the transition from hadronic to partonic matter, e.g. a strongly-coupled Quark-Gluon-Plasma (sQGP). In the present work, we report new results on the charmonium nuclear modification factor $R_{A A}$, rapidity distribution, the elliptic flow $v_{2}$ of $D$ mesons, the ratios $\langle J / \Psi\rangle /\langle\pi\rangle$ and $\Psi^{\prime} /(J / \Psi)$ for energies from about $20 \mathrm{~A} \cdot \mathrm{GeV}$ - relevant for the future Facility-for-Antiproton-and-Ion-Research (FAIR) - up to top RHIC energies.

We recall that in the early stage of the nucleus-nucleus collisions the dissociation and the regeneration of $J / \Psi$ by fundamentally different mechanisms are possible: The $c \bar{c}$ pairs produced early in the reaction - by gluon-gluon fusion in primary nucleon-nucleon interactions - might be completely dissociated in the dense medium and not be formed as bound states due to color screening. In this model scenario charmonia have to be recreated by some mechanism to yield a finite production cross section of $J / \Psi$ and $\Psi^{\prime}$. The $c \bar{c}$ pairs might also be formed in some pre-hadronic resonance (color-dipole) state that will further develop to the charmonium eigenstates in vacuum. Such resonance states can be dissociated in the medium due to interactions with other degrees of freedom but also be recreated by the inverse reaction channels. Independently, charmonia might also be generated in a statistical fashion at the phase boundary between the QGP and an interacting hadron gas such that their abundance would appear in statistical (chemical) equilibrium with the light and strange hadrons [12]. In the latter model the charmonium spectra carry no information on a possible preceeding partonic phase. Indeed, in Ref. [3] a success of the statistical hadronization model [4,5] has been put forward. Another alternative is the model for coalescence of charmonium in the SQGP [6]. For further variants or model concepts for charmonium suppression/enhancement we refer the reader to the reviews [7,8]. In this work our aim is to shed some light on various model concepts by exploiting relativistic microscopic transport theory.

The Hadron-String-Dynamics (HSD) approach [9] provides the space-time geometry of nucleus-nucleus reactions and a rather reliable estimate for the lo- 
cal energy densities achieved, since the production of secondary particles with light and single strange quarks/antquarks is described well from SIS to RHIC energies [10]. As we will show in Section 2, the high energy-densities reached in $A u+A u$ collisions at RHIC clearly indicate that a strongly interacting QGP (sQGP) has been created for a couple of $\mathrm{fm} / \mathrm{c}$ in the central overlap volume. However, a proper understanding of the transport properties of the partonic phase is still lacking. Presently the effective degrees of freedom in the sQGP are much debated and charmonia are a unique and promising probe that is sensitive to the properties of the early (and so far unknown) medium.

In the present systematic study we first test the HSD results for charmonium production in $p+p$ and $d+A u$ reactions at RHIC energies in comparison to the recent data. This is crucial in order to obtain an accurate baseline for the study of any anomalous suppression of charmonia in nucleus-nucleus collisions (see Sections 3 and 44). The interactions of $J / \Psi$ 's with mesons in the late stages of the collision (when the energy density falls below a critical value of about $1 \mathrm{GeV} / \mathrm{fm}^{3}$ corresponding roughly to the critical energy density for a parton/hadron phase transition) gives a sizable contribution to its anomalous suppression at all beam energies as demonstrated in Refs. [11,12,13,14,15]. Accordingly, this more obvious 'hadronic' contribution has to be incorporated when comparing possible models for QGP-induced charmonium suppression to experimental data. On the other hand, as known from our studies in Refs. [11,12] charmonium interactions with the purely hadronic medium alone (which is modeled rather precisely by HSD) are not sufficient to describe the $J / \Psi$ suppression pattern at RHIC in detail.

Based on the microscopic HSD transport theory, we investigate in particular the following scenarios for the anomalous absorption of charmonia:

(1) the 'threshold melting' mechanism;

(2) a dissociation by the scattering on hadron-like correlators, i.e. the 'comover' scenario;

(3) additional scattering of charm with pre-hadrons which might be considered as color neutral precursors of hadronic states (cf. Refs. [16,17,18,19]).

All implemented scenarios will be described in detail in Section 5. In Section 6 we will investigate in particular the effect of the interactions of charm quarks in the pre-hadronic medium on $R_{A A}(y)$ of $J / \Psi$ by comparing our calculations to RHIC data. To complete our study, we will provide excitation functions for the $J / \Psi$ survival probability $S(J / \Psi)$ and the ratios $B_{\mu \mu} \sigma(J / \Psi) / B_{\mu \mu}^{\prime} \sigma\left(\Psi^{\prime}\right)$ in Section [7. Furthermore, by studying the $J / \Psi$ to $\pi$ ratio as a function of the number of participating nucleons $N_{\text {part }}$, we will test the assumption of charmonium production by statistical hadronization as advocated in Refs. 2, 3, 20] (subsection 7.2) . A summary of results as well as a discussion of open problems will close our study in Section 8. 


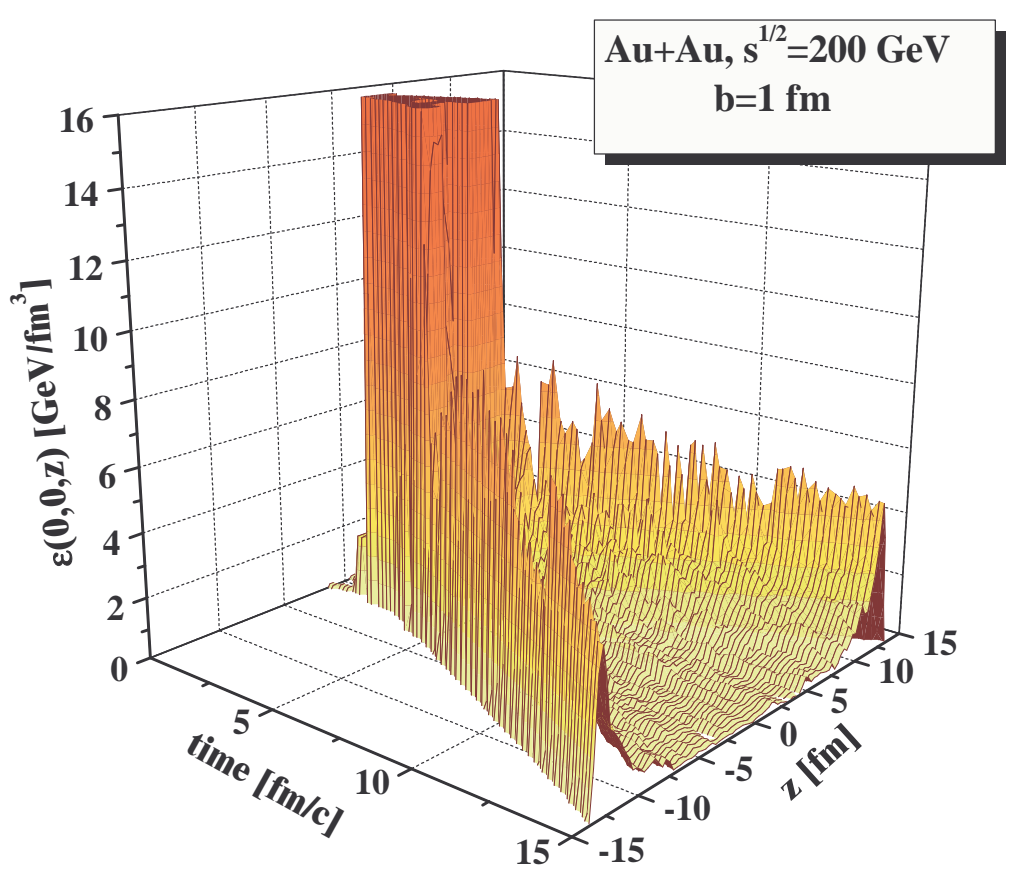

Fig. 1. The energy density $\varepsilon(x=0, y=0, z ; t)$ from HSD for a central $\mathrm{Au}+\mathrm{Au}$ collision at $\sqrt{s}=200 \mathrm{GeV}$. The time $t$ is given in the nucleon-nucleon center-of-mass system.

\section{$2 \quad$ Energy density}

The HSD transport model - employed in a large variety of $\pi+A, p+A, d+A$ and $A+A$ reactions - allows to calculate the energy-momentum tensor $T_{\mu \nu}(x)$ for all space-time points $x$ and thus the energy density $\varepsilon(x)=T_{00}(x)$ in the local rest frame. In order to exclude contributions to $T_{\mu \nu}$ from noninteracting nucleons in the initial phase all nucleons without prior interactions are discarded in the rapidity intervals $\left[y_{t a r}-0.4, y_{\text {tar }}+0.4\right]$ and $\left[y_{\text {pro }}-0.4, y_{\text {pro }}+0.4\right]$ where $y_{\text {tar }}$ and $y_{\text {pro }}$ denote projectile and target rapidity, respectively. Note that the initial rapidity distributions of projectile and target nucleons are smeared out due to Fermi motion by about \pm 0.4 . Some comments on the choice of the grid in space-time are in order here: In the actual calculation (for $\mathrm{Au}+\mathrm{Au}$ collisions) the initial grid has a dimension of $1 \mathrm{fm} \times 1 \mathrm{fm} \times 1 / \gamma_{c m} \mathrm{fm}$, where $\gamma_{c m}$ denotes the Lorentz $\gamma$-factor in the nucleon-nucleon center-of-mass system. After the time of maximum overlap $t_{m}$ of the nuclei the grid-size in beam direction $\Delta z_{0}=1 / \gamma_{c m}[\mathrm{fm}]$ is increased linearly in time as $\Delta z=\Delta z_{0}+a\left(t-t_{m}\right)$, where the parameter $a$ is chosen in a way to keep the particle number in the local cells of volume $\Delta V(t)=\Delta x \Delta y \Delta z(t)$ roughly constant during the longitudinal expansion of the system. In this way local fluctuations of the energy density $\varepsilon(x)$ due to fluctuations in the particle number are kept low. Furthermore, the time-step is taken as $\Delta t=0.2 \Delta z(t)$ and increases in time in analogy to 
$\Delta z(t)$. This choice provides a high resolution in space and time for the initial phase and keeps track of the relevant dynamics throughout the entire collision history.

The energy density $\varepsilon(\mathbf{r} ; t)$ - which is identified with the matrix element $T_{00}(\mathbf{r} ; t)$ of the energy momentum tensor in the local rest frame at space-time $(\mathbf{r}, t)-$ becomes very high in a central $\mathrm{Au}+\mathrm{Au}$ collision at $\sqrt{s}=200 \mathrm{GeV}$ as shown in Fig.1 ( $c f$. Fig. 1 of [11] for the corresponding energy density evolution in case of central collisions at top SPS energies). Fig. 1 shows the space-time evolution of the energy density $\varepsilon(x=0, y=0, z ; t)$ for a $\mathrm{Au}+\mathrm{Au}$ collision at $21300 \mathrm{AGeV}$ or $\sqrt{s}=200 \mathrm{GeV}$. It is clearly seen that energy densities above $16 \mathrm{GeV} / \mathrm{fm}^{3}$ are reached in the early overlap phase of the reaction and that $\varepsilon(x)$ drops after about $6 \mathrm{fm} / \mathrm{c}$ (starting from contact) below $1 \mathrm{GeV} / \mathrm{fm}^{3}$ in the center of the grid. On the other hand the energy density in the region of the leading particles - moving almost with the velocity of light - stays above $1 \mathrm{GeV} / \mathrm{fm}^{3}$ due to Lorentz time dilatation since the time $t$ in the transport calculation is measured in the nucleon-nucleon center-of-mass system. Note that in the local rest frame of the leading particles the eigentime $\tau$ is roughly given by $\tau \approx t / \gamma_{c m}$. As seen from Fig. 1, the energy density in the local rest frame is a rapidly changing function of time in nucleus-nucleus collisions. For orientation let us recall the relevant time scales (in the cms reference frame):

- The $c \bar{c}$ formation time $\tau_{c} \approx 1 / M_{\perp}$ is about $0.05 \mathrm{fm} / \mathrm{c}$ for a transverse mass of $4 \mathrm{GeV}$; the transient time for a central $\mathrm{Au}+\mathrm{Au}$ collision at $\sqrt{s}=200 \mathrm{GeV}$ is $t_{r} \approx 2 R_{A} / \gamma_{c m} \approx 0.13 \mathrm{fm} / \mathrm{c}$. According to standard assumptions, the $c \bar{c}$ pairs are produced in the initial hard $N N$ collisions dominantly by gluon fusion in the time period $t_{r}$. In fact, the formation time $\tau_{c}$ is significantly smaller than $t_{r}$, which implies the $c$ or $\bar{c}$ quarks may interact with the impinging nucleons of the projectile or target for times $t \leq t_{r}$.

- Using the Borken estimate for the energy density and employing the timescale $t_{r}=0.13 \mathrm{fm} / \mathrm{c}$, the energy density - after the nuclei have punched through each other - amounts to about $5 / 0.13>30 \mathrm{GeV} / \mathrm{fm}^{3}$ as quoted also in the HSD calculations in Ref. [11. Even when adding the $c \bar{c}$ formation time, this gives an energy density $\sim 5 / 0.18 \approx 28 \mathrm{GeV} / \mathrm{fm}^{3}$. So these numbers agree with transparent and simple estimates (cf. Fig. 1) and illustrate the high initial densities after $c \bar{c}$ production from primary interactions.

The energy densities quoted above are considerably different from the Bjorken estimate

$$
\tau \cdot \epsilon_{B j}=\frac{<E_{T}>\frac{d N}{d \eta}}{\pi R_{T}^{2}},
$$

where $<E_{T}>$ is the average transverse energy per particle, $d N / d \eta$ the number 

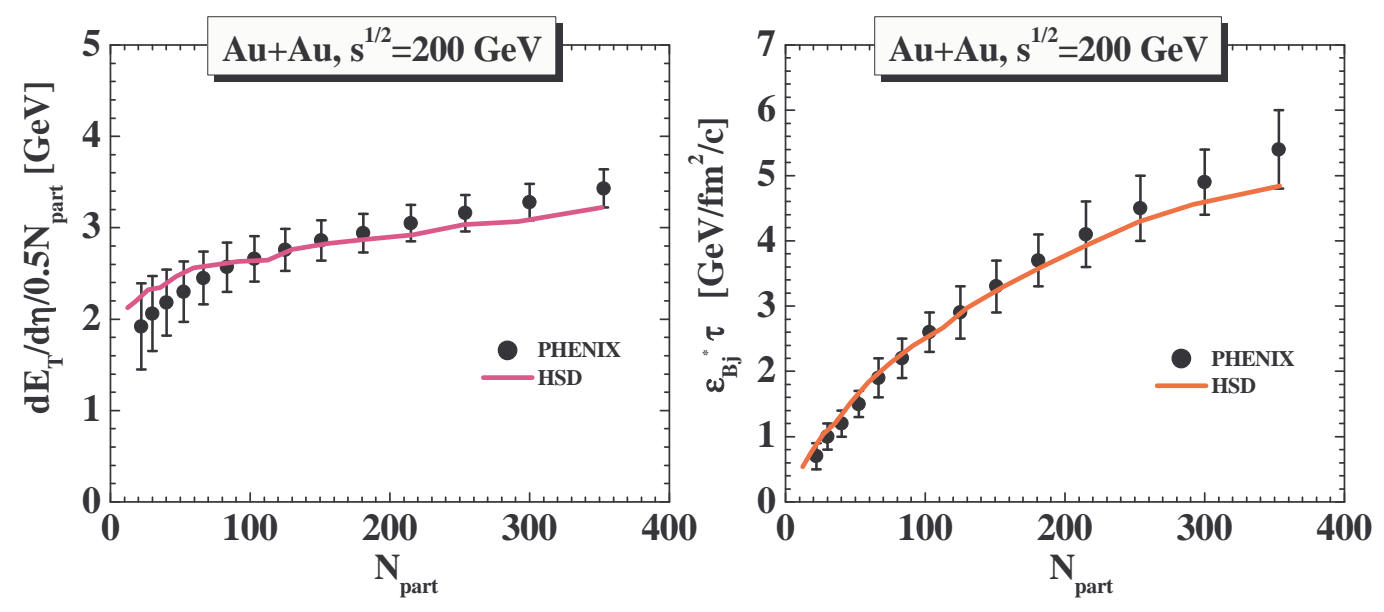

Fig. 2. Left part: The energy density $E_{T}$ per pseudorapidity interval $d \eta$ divided by the number of participant pairs $\left(0.5 N_{\text {part }}\right)$ from HSD (solid line) in comparison to the PHENIX data (dots) [21]. Right part: The Bjorken energy density $\varepsilon_{B j} \cdot \tau$ from HSD (solid line) for $\mathrm{Au}+\mathrm{Au}$ collisions at $\sqrt{s}=200 \mathrm{GeV}$ in comparison to the PHENIX data (dots) [21].

of particles per unit of pseudorapidity, and $\tau$ a formation time parameter often used as $\tau=1 \mathrm{fm} / \mathrm{c}$. Furthermore, $\pi R_{T}^{2}$ denotes the overlap area for the corresponding centrality. Is is important to point out that the estimate (1) is only well defined for the product $\tau \epsilon_{B j}$ ! The question naturally arises, if the transport calculations follow the corresponding experimental constraints.

To this aim we show $d E_{T} / d \eta$ (divided by half the number of participants $N_{\text {part }}$ ) from HSD 1 (l.h.s.) in comparison to the measurements by PHENIX [21]. Accordingly, the Bjorken energy density $\epsilon_{B j}-$ multiplied by the time-scale $\tau$ (11)from HSD is shown additionally in the r.h.s. in comparison to the PHENIX measurements as a function of $N_{\text {part }}$. The similarity between the calculated quantities and the experimental data demonstrates that the space-time evolution of the energy-momentum tensor $T_{\mu \nu}$ in HSD is sufficiently well under control. We now may step on with the actual investigation of the charmonium dynamics.

\section{Implementation of charmonium production}

In order to examine the dynamics of open charm and charmonium degrees of freedom during the formation and expansion phase of the highly excited system created in a relativistic nucleus-nucleus collision, one has to know the number of initially produced particles with $c$ or $\bar{c}$ quarks, i.e. $D, \bar{D}, D^{*}$, $\bar{D}^{*}, D_{s}, \bar{D}_{s}, D_{s}^{*}, \bar{D}_{s}^{*}, J / \Psi(1 S), \Psi^{\prime}(2 S), \chi_{c}(1 P)$. In this work we follow the previ-

$\overline{1}$ The open source code is available from Ref. [22] 


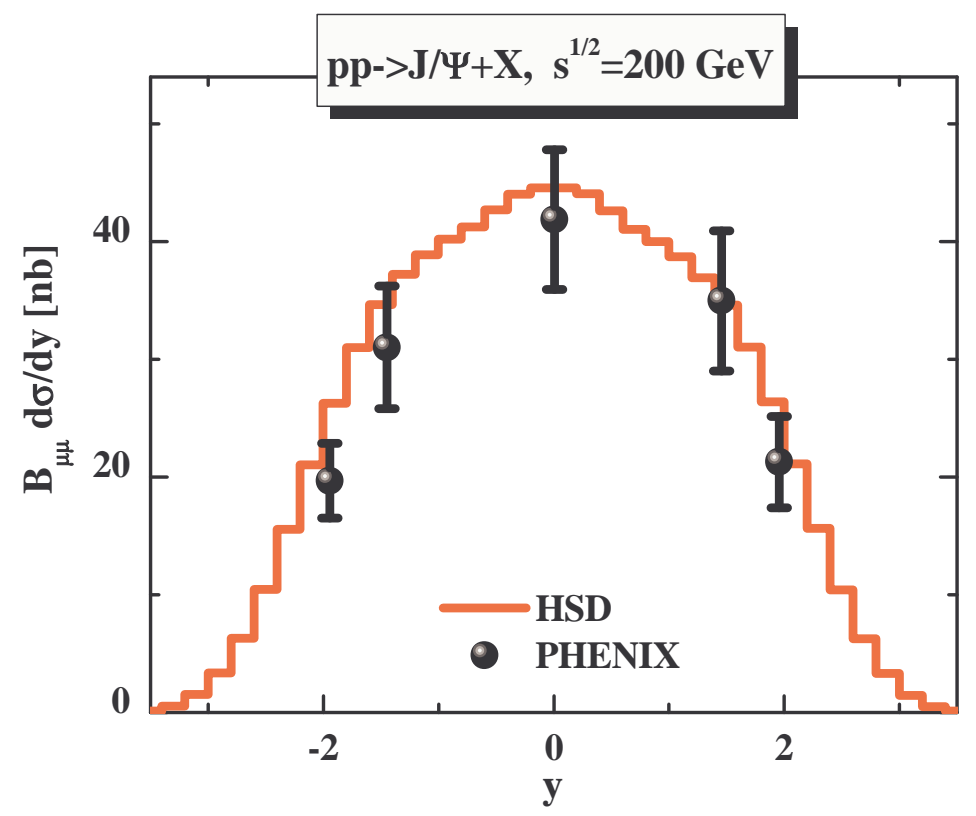

Fig. 3. Cross section for the differential $J / \Psi$ production in rapidity (times the branching ratio to di-muons $B_{\mu \mu}$ ) in $p p$ collisions at $\sqrt{s}=200 \mathrm{GeV}$. The HSD (input) parametrization (solid line) is compared to the PHENIX data (symbols) from Ref. [23].

ous studies in Refs. [9,11,13,14,24] and fit the total charmonium cross sections $\left(i=\chi_{c}, J / \Psi, \Psi^{\prime}\right)$ from $N N$ collisions as a function of the invariant energy $\sqrt{s}$ by the expression [12]

$$
\sigma_{i}^{N N}(s)=f_{i} a\left(1-\frac{m_{i}}{\sqrt{s}}\right)^{\alpha}\left(\frac{\sqrt{s}}{m_{i}}\right)^{\beta} \theta\left(\sqrt{s}-\sqrt{s_{0 i}}\right),
$$

where $m_{i}$ denotes the mass of charmonium $i$ while $\sqrt{s_{0 i}}=m_{i}+2 m_{N}$ is the threshold in vacuum. The parameters in (2) have been fixed to describe the $J / \Psi$ and $\Psi^{\prime}$ data up to the RHIC energy $\sqrt{s}=200 \mathrm{GeV}$ ( $c f$. [11]). We use $a=0.16 \mathrm{mb}, \alpha=10, \beta=0.775$. Note, that for the present study we updated our parametrization (i.e. reduced the parameter $a$ by $\sim 20 \%$ ) according to the latest PHENIX data [23].

The parameters $f_{i}$ are fixed as $f_{\chi_{c}}=0.636, f_{J / \Psi}=0.581, f_{\Psi^{\prime}}=0.21$ in order to reproduce the experimental ratio

$$
\frac{B\left(\chi_{c 1} \rightarrow J / \Psi\right) \sigma_{\chi_{c 1}}+B\left(\chi_{c 2} \rightarrow J / \Psi\right) \sigma_{\chi_{c 2}}}{\sigma_{J / \Psi}^{\exp }}=0.344 \pm 0.031
$$

measured in $p p$ and $\pi N$ reactions [25,26] as well as the averaged $p p$ and $p A$ ratio $\left(B_{\mu \mu}\left(\Psi^{\prime}\right) \sigma_{\Psi^{\prime}}\right) /\left(B_{\mu \mu}(J / \Psi) \sigma_{J / \Psi}\right) \simeq 0.0165$ (cf. the compilation of experimental data in Ref. [27]). The experimentally measured $J / \Psi$ cross section 
includes the direct $J / \Psi$ component $\left(\sigma_{J / \Psi}\right)$ as well as the decays of higher charmonium states $\chi_{c}$ and $\Psi^{\prime}$, i.e.

$$
\sigma_{J / \Psi}^{e x p}=\sigma_{J / \Psi}+B\left(\chi_{c} \rightarrow J / \Psi\right) \sigma_{\chi_{c}}+B\left(\Psi^{\prime} \rightarrow J / \Psi\right) \sigma_{\Psi^{\prime}}
$$

Note, we do not distinguish the $\chi_{c 1}(1 P)$ and $\chi_{c 2}(1 P)$ states. Instead, we use only the $\chi_{c 1}(1 P)$ state (which we denote as $\chi_{c}$ ), however, with an increased branching ratio for the decay to $J / \Psi$ in order to include the contribution of $\chi_{c 2}(1 P)$, i.e. $B\left(\chi_{c} \rightarrow J / \Psi\right)=0.54$. Furthermore, we adopt $B\left(\Psi^{\prime} \rightarrow J / \Psi\right)=$ 0.557 from Ref. [28].

We recall that (as in Refs. [13,14,29,30,31]) the charm degrees of freedom in the HSD approach are treated perturbatively and that initial hard processes (such as $c \bar{c}$ or Drell-Yan production from $N N$ collisions) are 'pre-calculated' to achieve a scaling of the inclusive cross section with the number of projectile and target nucleons as $A_{P} \times A_{T}$ when integrating over impact parameter. For fixed impact parameter $b$, the $c \bar{c}$ yield then scales with the number of binary hard collisions $N_{\text {coll }}$ ( $c f$. Fig. 8 in Ref. [13]).

In addition to primary hard $N N$ collisions, the open charm mesons or charmonia may also be generated by secondary meson-baryon $(m B)$ reactions. Here we include all secondary collisions of mesons with baryons by assuming that the open charm cross section (from Section 2 of Ref. [13]) only depends on the invariant energy $\sqrt{s}$ and not on the explicit meson or baryon state. Furthermore, we take into account all interactions of 'formed' mesons - after a formation time of $\tau_{F}=0.8 \mathrm{fm} / \mathrm{c}$ (in their rest frame) 32 - with baryons or diquarks. For the total charmonium cross sections from meson-baryon (or $\pi N$ ) reactions we use the parametrization (in line with Ref. [33]):

$$
\sigma_{i}^{\pi N}(s)=f_{i} b\left(1-\frac{m_{i}}{\sqrt{s}}\right)^{\gamma}
$$

with $\gamma=7.3$ and $b=1.24 \mathrm{mb}$, which describes the existing experimental data at low $\sqrt{s}$ reasonably well, as seen in Ref. [11].

Apart from the total cross sections for charmonia we also need the differential distribution of the produced mesons in the transverse momentum $p_{T}$ and the rapidity $y$ (or Feynman $x_{F}$ ) from each individual collision. We recall that $x_{F}=p_{z} / p_{z}^{\max } \approx 2 p_{z} / \sqrt{s}$ with $p_{z}$ denoting the longitudinal momentum. For the differential distribution in $x_{F}$ from $N N$ and $\pi N$ collisions we use the ansatz from the E672/E706 Collaboration 34 and for the $p_{T}$ distribution a power low parametrization from Ref. [35] which has been fixed by the STAR 

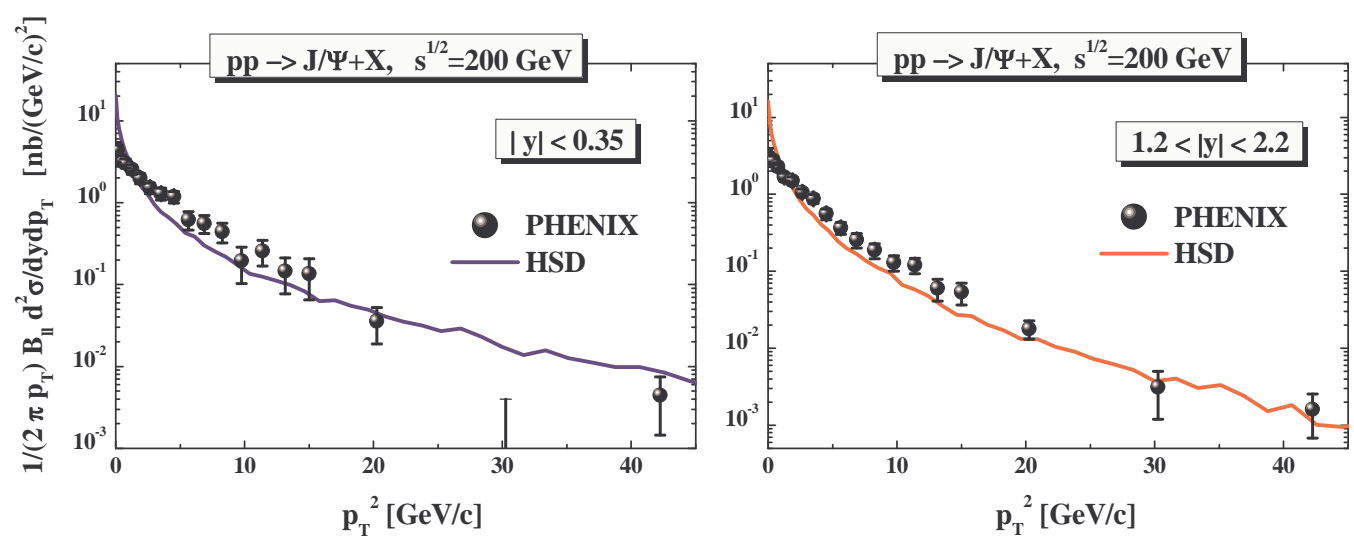

Fig. 4. Differential cross section of $J / \Psi$ production in $p p$ collisions at $\sqrt{s}=200 \mathrm{GeV}$ at mid-rapidity $(|y|<0.35$, l.h.s. $)$ and at forward rapidity $(1.2<|y|<2.2$, r.h.s. $)$ vs the transverse momentum squared $p_{T}^{2}$ as implemented in HSD (solid line) compared to the PHENIX data from Ref. [23] (dots).

data [36], i.e.

$$
\frac{d N}{d x_{F} d p_{T}} \sim\left(1-\left|x_{F}\right|\right)^{c}\left(1+\frac{p_{T}}{b_{p_{T}}}\right)^{c_{p_{T}}},
$$

with $b_{p_{T}}=3.5 \mathrm{GeV} / c$ and $c_{p_{T}}=-8.3$. The exponent $c$ is given by $c=$ $a /(1+b / \sqrt{s})$ and the parameters $a, b$ are chosen as $a_{N N}=16, b_{N N}=24.9$ $\mathrm{GeV}$ for $N N$ collisions and $a_{\pi N}=4.11, b_{\pi N}=10.2 \mathrm{GeV}$ for $\pi N$ collisions.

The resulting rapidity distribution for $J / \Psi$ production in $p p$ collisions at $\sqrt{s}=$ $200 \mathrm{GeV}$ is shown in Fig. 3 which is in line with the data from Ref. [23] within error bars. We also present the $p p \rightarrow J / \Psi+X$ differential cross section in $p_{T}^{2}$ at mid-rapidity $(|y|<0.35)$ and at forward rapidity (averaged in the interval $1.2<|y|<2.2)$ in Fig. 4. The HSD parametrization is compared to the recent measurements of the corresponding quantities by PHENIX [23]. The total cross sections for $D+\bar{D}$ production in this study are the same as those presented in Ref. [11].

The parametrizations of the total and differential cross sections for open charm mesons from $p N$ and $\pi N$ collisions are taken as in Refs. [13.14], apart from a readjustment of the parameter $a_{N N}$ in order to reproduce the recently measured rapidity distribution of $J / \Psi$ in $p+p$ reactions at $\sqrt{s}=200 \mathrm{GeV}$ by PHENIX [23].

For $D, D^{*}, \bar{D}, \bar{D}^{*}$ - meson $(\pi, \eta, \rho, \omega)$ scattering we refer to the calculations from Ref. [37,38] which predict elastic cross sections in the range of 10-20 mb depending on the size of the form factor employed. As a guideline we use a constant cross section of $10 \mathrm{mb}$ for elastic scattering with formed mesons and also baryons, although the latter might be even higher for very low relative 


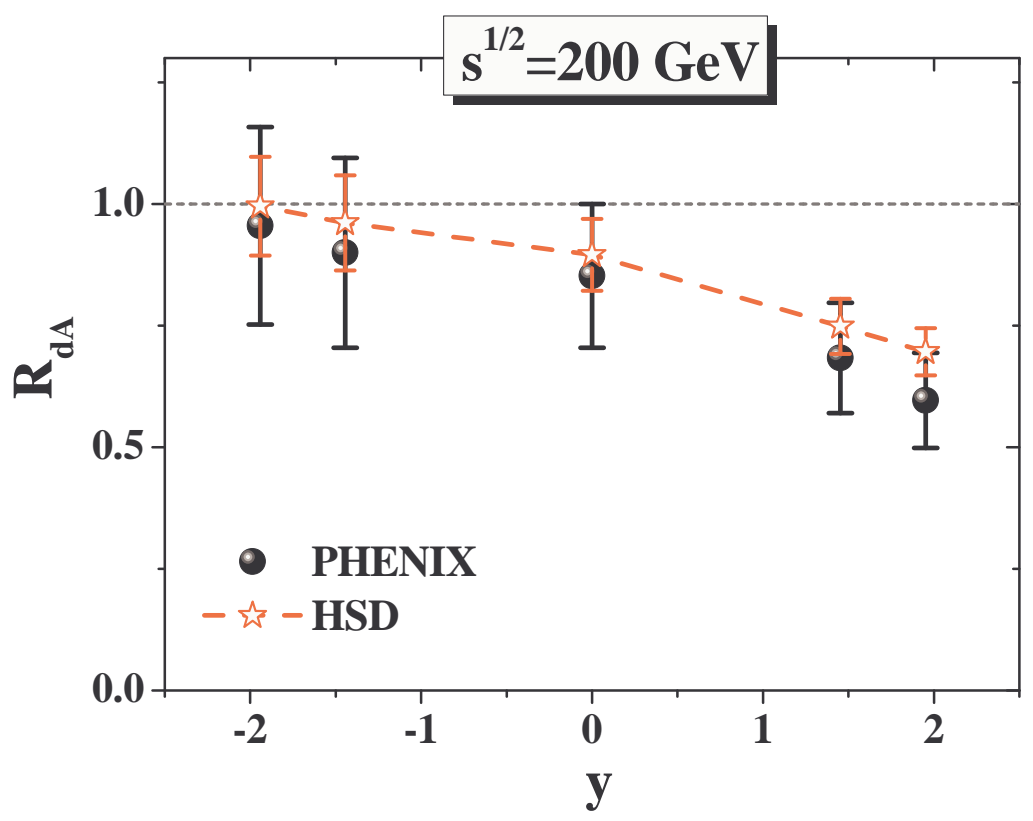

Fig. 5. $J / \Psi$ production cross section in $d+A u$ collisions relative to that in $p+p$ collisions (see text for the definition of $R_{d A}$ ) in HSD (red stars) as compared to the PHENIX data 39] (full dots).

momenta. We will discuss this issue in more detail in Section 5.1.

\section{Baryonic ('normal') nuclear absorption}

The yield of $J / \Psi$ in $p+A$ and $A+A$ reactions is modified compared to that in $p+p$ scaled with the number of initial binary scatterings $N_{\text {coll }}$ [40]41]. Indeed, the produced $c \bar{c}$ can be dissociated or absorbed on either the residual nucleus of the projectile or target or on light co-moving particles (usually on mesons or, at high energy, on partons) produced in the very early phase. The latter reactions are only important in nucleus-nucleus collisions and not in $p+A$ or $d+A$ as the number of 'comovers' created in proton- or deuteron-induced processes is small. In contrast, charmonium absorption on baryons is the leading suppression mechanism in $d+A(p+A)$ scattering and is an important base-line for the study of the absorption in the hot and dense medium created in $A+A$ reactions.

In order to study the effect of charmonium rescattering on projectile/target nucleons, we adopt in HSD the following dissociation cross sections of charmonia with baryons independent of the energy:

$$
\begin{aligned}
& \sigma_{c \bar{c} B}=4.18 \mathrm{mb} ; \\
& \sigma_{J / \Psi B}=4.18 \mathrm{mb} ; \sigma_{\chi_{c} B}=4.18 \mathrm{mb} ; \sigma_{\Psi^{\prime} B}=7.6 \mathrm{mb} .
\end{aligned}
$$


In (66) the cross section $\sigma_{c \bar{c} B}$ stands for a (color dipole) pre-resonance $(c \bar{c})$ baryon cross section, since the $c \bar{c}$ pair produced initially cannot be identified with a particular charmonium due to the uncertainty relation in energy and time. For the life-time of the pre-resonance $c \bar{c}$ pair (in it's rest frame) a value of $\tau_{c \bar{c}}=0.3 \mathrm{fm} / \mathrm{c}$ is assumed following Ref. [42]. This time scale corresponds to the mass difference of the $\Psi^{\prime}$ and $J / \Psi$.

The values for the cross sections $\sigma_{J / \Psi N}, \sigma_{c \bar{c} N}$ at RHIC energies are currently debated in the literature. On one side, all the data on the $J / \Psi$ production in $p+A$ at energies $\sqrt{s} \leq 40 \mathrm{GeV}$ were found to be consistent with an energyindependent cross section of the order of $4-7 \mathrm{mb}$ [40,43,44,45,46]. On the other hand, the corresponding cross sections at the much higher energy of $\sqrt{s}=200 \mathrm{GeV}$, e.g. at RHIC, are expected to be smaller [47], since part of the suppression might be attributed to other (initial-state) cold-matter effects, such as gluon shadowing [48,49,50, radiative gluon energy loss in the initial state or multiple gluon rescattering. We recall that 'shadowing' is a depletion of low-momentum partons in a nucleon embedded in a nucleus compared to the population in a free nucleon, which leads to a lowering in the charmonium production cross section. The reasons for depletion, though, are numerous, and models of shadowing vary accordingly. There is, therefore, a considerable (about a factor of 3) uncertainty in the amount of shadowing predicted at RHIC [48,49,50,51,52]. In the analysis of the $d+A u$ data at $\sqrt{s}=200 \mathrm{GeV}$, in which the maximum estimate for the effect of the shadowing was made [47,50], the additional absorption on baryons allowed by the data was found to lead to $\sigma_{J / \Psi N}=1-3 \mathrm{mb}$ or higher, if some contribution of anti-shadowing is present. The authors of [50] advocate $\sigma_{J / \Psi N}=3 \mathrm{mb}$ in order to preserve the agreement with the data of the Fermilab experiment E866. The PHENIX Collaboration [39] finds a breakup cross section of $2.8_{-1.4}^{+1.7} \mathrm{mb}$ (using EKS shadowing) which still overlaps with the CERN value of $4.18 \mathrm{mb}$ (though with large error bars). However, the theoretical uncertainty is still large, since in the works above only an approximate model for baryonic absorption was applied and not a microscopic transport approach that e.g. also includes secondary production channels of charm pairs as described in Section 3.

Within HSD we have found the baryoninc absorption cross sections (6) to agree with the data at SPS energies [11]. In Fig. 5 we compare the HSD result (employing the same cross sections (6) for baryonic absorption and neglecting shadowing) for the $J / \Psi$ production in $d+A u$ collisions at $\sqrt{s}=200 \mathrm{GeV}$ to the inclusive PHENIX data [39]. The quantity plotted is the nuclear modification factor defined as

$$
R_{d A} \equiv \frac{d N_{J / \Psi}^{d A u} / d y}{\left\langle N_{\text {coll }}\right\rangle \cdot d N_{J / \Psi}^{p p} / d y}
$$



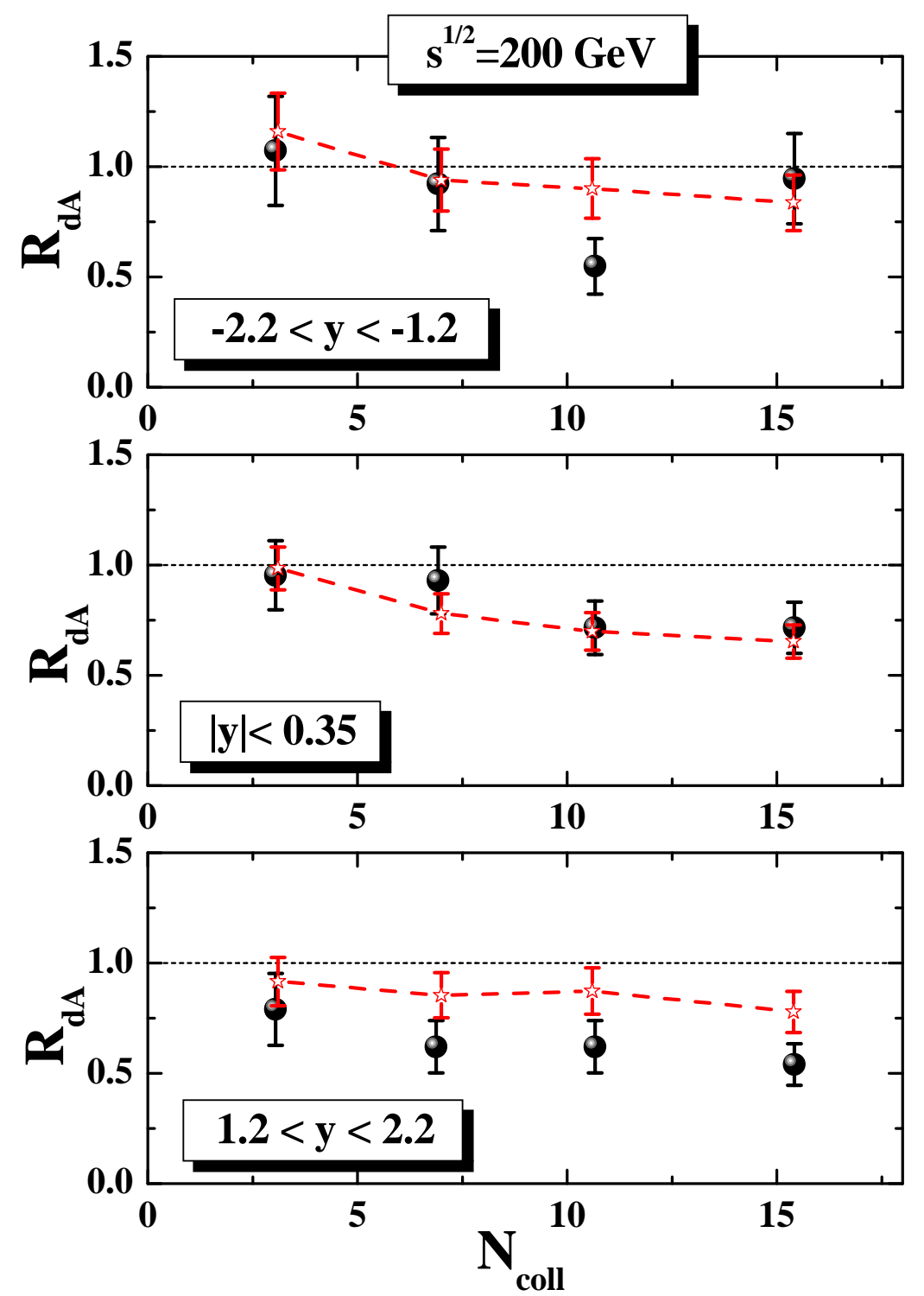

Fig. 6. The ratio $R_{d A}$ (77) for backward, central and forward rapidity bins as a function of the number of binary collisions $N_{\text {coll }}$ for $d+A u$ at $\sqrt{s}=200 \mathrm{GeV}$. The experimental data have been taken from Ref. [39]. The HSD results (stars connected by red dashed lines) show calculations without including low- $x$ gluon shadowing and slightly overestimate $R_{d A}$ in the forward interval $1.2<y<2.2$. The theoretical error bars are due to the finite statistics of the calculation.

where $d N_{J / \Psi}^{d A u} / d y$ is the $J / \Psi$ invariant yield in $d+A$ collisions, $d N_{J / \Psi}^{p p} / d y$ is the $J / \Psi$ invariant yield in $p+p$ collisions; $\left\langle N_{\text {coll }}\right\rangle$ is the average number of binary collisions for the same rapidity bin. In our analysis we have used $\left\langle N_{\text {coll }}\right\rangle=7.6 \pm 0.3$ according to the PHENIX estimate [39].

It is seen from Fig. 5 that the calculations follow approximately the decrease in $R_{d A}$ with rapidity, however, with a tendency to overshoot at forward rapidity. Within error bars we find the values of $\sigma_{c \bar{c} B}$ from (6) to be compatible with 
the inclusive RHIC measurement as well as with the lower energy data [44]. This finding is also in line with the analysis of the PHENIX Collaboration in Ref. 39]

In order to shed some further light on the role of shadowing, we compare our calculations for $R_{d A}$ in different rapidity bins as a function of the centrality of the $d+A u$ collision, which in Fig. [6 is represented by the number of binary collisions $N_{\text {coll }}$. The latter number is directly taken from the number of binary hard $N N$ collisions in the transport calculation while the comparison with experiment is based on a Glauber model analysis of the data similar to that performed in Ref. [53]. The actual results displayed in Fig. 6 (stars connected by dashed lines) and the PHENIX data from Ref. [39] are roughly compatible for the rapidity intervals $-2.2<y<-1.2$ and $|y|<0.35$, but demonstrate that the suppression at forward rapidity $(1.2<y<2.2)$ is underestimated in the color-dipole dissociation model with a constant cross section of $4.18 \mathrm{mb}$. This clearly points to the presence of shadowing effects at least at forward rapidities which is not so pronounced in the inclusive data set in Fig. 5. A more serious question is a quantification of the shadowing due to the limited statistics of both the experimental data and the calculations. Here we do not attempt to attribute a fixed number for the shadowing effect but merely point out that independent high statistics data will be necessary to fix this unsatisfactory situation from the experimental side.

Nevertheless, some note of caution is appropriate for the further analysis of charmonium suppression in $A u+A u$ collisions: There are 'cold nuclear matter effects' such as 'gluon shadowing' beyond those incorporated in the transport calculations, and especially quantitative statements about any 'agreement with data' might have to be reconsidered. In case of $A u+A u$ reactions the shadowing from projectile/target will show up symmetrically around $y=0$ and in part contribute to the stronger $J / \Psi$ suppression at forward/backward rapidities. Nevertheless, following Granier de Cassagnac [53], an anomalous suppression of $J / \Psi$ beyond 'cold nuclear matter effects' is clearly present in the $A u+A u$ data to be investigated below.

\section{Scenarios for the anomalous $J / \Psi$ suppression}

It is well known that the baryonic (normal) absorption alone cannot explain the suppression of charmonia in heavy-ion collisions with increasing centrality [7]. We have implemented in HSD several different mechanism for the additional (anomalous) suppression of charmonia which will be explained in the following Subsections. By comparing the results from these scenarios to each other and to the available data the mechanism of charmonium interactions with the medium can be probed. 


\section{1 'Comover' suppression (and recombination)}

First of all let us stress that the interactions with 'comoving' mesons lead not only to the dissociation of charmonia, but also to their recreation via the inverse recombination process $D+\bar{D} \rightarrow c \bar{c}+m$, where $m=\{\pi, \rho, \omega, K, \ldots\}$. As already pointed out before, the $J / \Psi, \chi_{c}, \Psi^{\prime}$ formation cross sections by open charm mesons or the inverse 'comover' dissociation cross sections are not well known and the significance of these channels is discussed controversely in the literature [4,6,54,55,56,57,58]. We here follow the concept of Refs. [14,15] and introduce a simple 2-body transition model with a single parameter $\left|M_{0}\right|^{2}$, that allows to implement the backward reactions uniquely by employing detailed balance for each individual channel.

Since the charmonium-meson dissociation and backward reactions typically occur with low relative momenta ('comovers'), it is legitimate to write the cross section for the process $1+2 \rightarrow 3+4$ as

$$
\sigma_{1+2 \rightarrow 3+4}(s)=2^{4} \frac{E_{1} E_{2} E_{3} E_{4}}{s}\left|\tilde{M}_{i}\right|^{2}\left(\frac{m_{3}+m_{4}}{\sqrt{s}}\right)^{6} \frac{p_{f}}{p_{i}},
$$

where $E_{k}$ denotes the energy of hadron $k(k=1,2,3,4)$, respectively. The initial and final momenta for fixed invariant energy $\sqrt{s}$ are given by

$$
\begin{aligned}
& p_{i}^{2}=\frac{\left(s-\left(m_{1}+m_{2}\right)^{2}\right)\left(s-\left(m_{1}-m_{2}\right)^{2}\right)}{4 s}, \\
& p_{f}^{2}=\frac{\left(s-\left(m_{3}+m_{4}\right)^{2}\right)\left(s-\left(m_{3}-m_{4}\right)^{2}\right)}{4 s},
\end{aligned}
$$

where $m_{k}$ denotes the mass of hadron $k$. In (8) $\left|\tilde{M}_{i}\right|^{2}\left(i=\chi_{c}, J / \Psi, \Psi^{\prime}\right)$ stands for the effective matrix element squared, which for the different 2-body channels is taken of the form

$$
\begin{aligned}
& \left|\tilde{M}_{i}\right|^{2}=\left|M_{i}\right|^{2} \text { for }(\pi, \rho)+(c \bar{c})_{i} \rightarrow D+\bar{D} \\
& \left|\tilde{M}_{i}\right|^{2}=3\left|M_{i}\right|^{2} \text { for }(\pi, \rho)+(c \bar{c})_{i} \rightarrow D^{*}+\bar{D}, D+\bar{D}^{*}, D^{*}+\bar{D}^{*} \\
& \left|\tilde{M}_{i}\right|^{2}=\frac{1}{3}\left|M_{i}\right|^{2} \text { for }\left(K, K^{*}\right)+(c \bar{c})_{i} \rightarrow D_{s}+\bar{D}, \bar{D}_{s}+D \\
& \left|\tilde{M}_{i}\right|^{2}=\left|M_{i}\right|^{2} \text { for }\left(K, K^{*}\right)+(c \bar{c})_{i} \rightarrow D_{s}+\bar{D}^{*}, \bar{D}_{s}+D^{*}, D_{s}^{*}+\bar{D} \\
& \bar{D}_{s}^{*}+D, \bar{D}_{s}^{*}+D^{*}
\end{aligned}
$$

The relative factors of 3 in (10) are guided by the sum rule studies in [59] which suggest that the cross section is increased whenever a vector meson $D^{*}$ or $\bar{D}^{*}$ appears in the final channel while another factor of $1 / 3$ is introduced 
for each $s$ or $\bar{s}$ quark involved. The factor $\left(\left(m_{3}+m_{4}\right) / \sqrt{s}\right)^{6}$ in (8) accounts for the suppression of binary channels with increasing $\sqrt{s}$ and has been fitted to the experimental data for the reactions $\pi+N \rightarrow \rho+N, \omega+N, \phi+N, K^{+}+\Lambda$ in Ref. 60.

We use the same matrix elements for the dissociation of all charmonium states $i\left(i=\chi_{c}, J / \Psi, \Psi^{\prime}\right)$ with mesons:

$$
\left|M_{J / \Psi}\right|^{2}=\left|M_{\chi_{c}}\right|^{2}=\left|M_{\Psi^{\prime}}\right|^{2}=\left|M_{0}\right|^{2}
$$

We note for completeness that in Ref. 14 the parameter $\left|M_{0}\right|^{2}$ was fixed by comparison to the $J / \Psi$ suppression data from the NA38 and NA50 Collaborations for $\mathrm{S}+\mathrm{U}$ and $\mathrm{Pb}+\mathrm{Pb}$ collisions at 200 and $158 \mathrm{AGeV}$, respectively. In a later study [11], however, this parameter has been readjusted in accordance with the updated value of the cross section (6) of charmonium dissociation on baryons (following the latest NA50 and NA60 analysis [40,43]). The best fit is obtained for $\left|M_{0}\right|^{2}=0.18 \mathrm{fm}^{2} / \mathrm{GeV}^{2}$; this value will be employed in our following studies, too.

The advantage of the model introduced in [14,15] is that detailed balance for the binary reactions can be employed strictly for each individual channel, i.e.

$$
\sigma_{3+4 \rightarrow 1+2}(s)=\sigma_{1+2 \rightarrow 3+4}(s) \frac{\left(2 S_{1}+1\right)\left(2 S_{2}+1\right)}{\left(2 S_{3}+1\right)\left(2 S_{4}+1\right)} \frac{p_{i}^{2}}{p_{f}^{2}}
$$

and the role of the backward reactions $\left((c \bar{c})_{i}+\right.$ meson formation by $D+\bar{D}$ flavor exchange) can be explored without introducing any additional parameter once $\left|M_{0}\right|^{2}$ is fixed. In Eq. (12) the quantities $S_{j}$ denote the spins of the particles, while $p_{i}^{2}$ and $p_{f}^{2}$ denote the cms momentum squared in the initial and final channels, respectively. The uncertainty in the cross sections (12) is of the same order of magnitude as that in Lagrangian approaches using e.g. $S U(4)_{\text {flavor }}$ symmetry [37,38, since the form factors at the vertices are essentially unknown [59]. It should be pointed out that the 'comover' dissociation channels for charmonia are described in HSD with the proper individual thresholds for each channel in contrast to the more schematic 'comover' absorption model in Refs. [52,61].

The regeneration of charmonia by recombination of $D\left(D^{*}\right)$ mesons in the hadronic phase was first studied by C.M. Ko and collaborators in [58]. The conclusion at that time was that this process is unlikely at RHIC energies [55, 58, 62]. On the other hand, it has been shown within HSD [14 that the contribution of the $D+\bar{D}$ annihilation to the produced $J / \Psi$ at RHIC is considerable. Moreover, the equilibrium in the reaction $J / \Psi+m \leftrightarrow D \bar{D}$ is reached (i.e. the charmonium recreation is comparable with the dissociation by 'comov- 
ing' mesons). The reason for such differences is that the pioneering study [58] within the hadron gas model was confined to $J / \Psi$ reactions with $\pi$ 's into two particular $D \bar{D}$ channels $\left(D+\bar{D}^{*}\right.$ and $\left.D^{*}+\bar{D}^{*}\right)$. On the contrary, in Ref. [14] the interactions with all mesons into all possible combinations of $D \bar{D}$ states have been taken into account. Note that the $\rho$-meson density at RHIC is large such that the channel with the most abundant $\rho$-meson resonance is dominant. Furthermore, in Ref. [14] the feed down from $\chi_{c}$ and $\Psi^{\prime}$ decays has been considered. The results of [14] are in accordance with independent studies in Refs. 63, 64, 65 66]. Later work within the HSD approach [12] has supported the conclusions of Ref. [14] and stressed the importance for $D \bar{D}$ annihilation in the late (purely hadronic) stages of the collisions.

\section{2 'Threshold melting'}

This scenario is based on the idea of sequential dissociation of charmonia with increasing temperature [67,68,69,70], i.e. of charmonium melting in the QGP due to color screening as soon as the fireball temperature reaches the dissociation temperatures of $\left(\approx 2 T_{c}\right.$ for $J / \Psi, \approx T_{c}$ for excited states, where $T_{c}$ stands for the critical temperature of the deconfinement phase transition). In the early approaches the temperature of the fireball has been estimated using e.g. the Bjorken formula (11). We modify the standard sequential dissociation model in two aspects: (i) the energy density is calculated locally and microscopically instead of using schematic estimates ( $c f$. section 2); (ii) the model incorporates a charmonium regeneration mechanism (by $D \bar{D}$ annihilation processes).

The 'threshold scenario' for charmonium dissociation now is implemented in a straight forward way: whenever the local energy density $\varepsilon(x)$ is above a threshold value $\varepsilon_{j}$, where the index $j$ stands for $J / \Psi, \chi_{c}, \Psi^{\prime}$, the charmonium is fully dissociated to $c+\bar{c}$. The default threshold energy densities adopted are

$$
\varepsilon_{J / \Psi}=16 \mathrm{GeV} / \mathrm{fm}^{3}, \varepsilon_{\chi_{c}}=2 \mathrm{GeV} / \mathrm{fm}^{3} \text {, and } \varepsilon_{\Psi^{\prime}}=2 \mathrm{GeV} / \mathrm{fm}^{3} \text {. }
$$

The dissociation of charmonia is widely studied using lattice QCD (lQCD) [71,72,73,74,75] in order to determine the dissociation temperature (or energy density) via the maximum entropy method. On the other hand one may use potential models - reproducing the charmonium excitation spectrum in vacuum - to calculate Mott transition temperatures in a hot medium. Both approaches have their limitations and the quantitative agreement between the different groups is still unsatisfactory:

- (A) Potential models employ the static heavy quark-antiquark pair free energy - calculated on the lattice - to obtain the charmonium spectral func- 
tions. This leads to the dissociation temperatures [76]

$$
T_{\text {melt }}(J / \Psi) \leq 1.2 T_{c}, T_{\text {melt }}\left(\chi_{c}\right) \leq T_{c}, T_{\text {melt }}\left(\Psi^{\prime}\right) \leq T_{c} .
$$

- (B) The maximum entropy method is used to relate the Euclidean thermal correlators of charmonia - calculated on the lattice - to the corresponding spectral functions and yields higher dissociation temperatures [71]

$$
T_{\text {melt }}(J / \Psi)=1.7-2 T_{c}, T_{\text {melt }}\left(\chi_{c}\right)=1.1-1.2 T_{c}
$$

or 72 ]

$$
T_{\text {melt }}(J / \Psi) \geq 1.5 T_{c}, T_{\text {melt }}\left(\chi_{c}\right)=1.1 T_{c} .
$$

Our earlier analysis of experimental data at the SPS in the 'threshold melting' approach [11] lead us to conclude from the observation of a considerable amount of $J / \Psi$ in the most central $P b+P b$ collisions that the assumption of a melting of $J / \Psi$ close to $T_{c}$ contradicts the data. Therefore, the values (13) are applied also in the current study.

\subsection{Discriminating hadronic and partonic phases}

Two more scenarios are implemented in our present HSD simulations that are closely related to the 'comover suppression' and the 'threshold melting' scenarios outlined in the previous sub-sections. The essential difference is that the comoving hadrons (including the $D$-mesons) exist only at energy densities below some energy density $\varepsilon_{c u t}$, which is a free parameter. We employ $\varepsilon_{c u t}=\varepsilon_{c} \approx 1 \mathrm{GeV} / \mathrm{fm}^{3}$, which is equal to the critical energy density $\varepsilon_{c}$ for the parton/hadron phase transition. This scenario clearly separates 'formed hadrons' from possible pre-hadronic states at higher energy densities. Indeed, it is currently not clear whether $D$ - or $D^{*}$-mesons survive at energy densities above $\varepsilon_{c}$ but hadronic correlators with the quantum numbers of the hadronic states are likely to persist above the phase transition [77. One may speculate that similar correlations (pre-hadrons) survive also in the light quark sector above $T_{c}$ such that 'hadronic comovers' - with modified spectral functions might show up also at energy densities above $\varepsilon_{c}$.

We recall that the concept of (color neutral) pre-hadrons - explained in more detail in Refs. [16]18 - has been also used in the hadron electroproduction studies off nuclei in Refs. [16[17] as well as for high $p_{T}$ hadron suppression [18] or jet suppression at RHIC energies [19]. It has been found that the pre-hadron concept works well for hadron attenuation in nuclei at HERMES energies [16]17] but underestimates the high $p_{T}$ hadron suppression [18] as well as the jet attenuation at RHIC energies [19]. Nevertheless, the amount of attenuation due to such pre-hadronic interactions emerged to be about $50 \%$ 
of the experimentally observed suppression at RHIC such that their effect might not simply be discarded. It should be stressed that the concept of prehadrons refers to the string breaking mechanism as described in Refs. [16,18] and is independent on the energy density. A detailed study on the space-time evolution of pre-hadrons and their formation to hadrons for $p p$ collisions has been performed by Gallmeister and Falter in Ref. [78].

In line with the investigations in Refs. [18,19] we also study $J / \Psi$ production and absorption in $A u+A u$ collisions at $\sqrt{s}=200 \mathrm{AGeV}$ assuming the absorption of charmonia on pre-hadrons as well as their regeneration by prehadrons. This adds additional interactions of the particles with charm quarks (antiquarks) in the very early phase of the nucleus-nucleus collisions as compared to the default HSD approach. Since these pre-hadronic (color-dipole) states represent some new degrees-of-freedom, the interactions of charmed states with these objects have to be specified separately.

For notation we define a pre-hadronic state consisting of a quark-antiquark pair as pre-meson $\tilde{m}$ and a state consisting of a diquark-quark pair as prebaryon $\tilde{B}$. The dissociation cross section of a $c \bar{c}$ color dipole state with a pre-baryon is taken to be of the same order as with a formed baryon,

$$
\sigma_{c \bar{c} \tilde{B}}^{\text {diss }}=5.8 \mathrm{mb}
$$

whereas the cross section with a pre-meson follows from the additive quark model as [16,17]

$$
\sigma_{c \bar{c} \tilde{m}}^{d i s s}=\frac{2}{3} \sigma_{c \bar{c} \tilde{B}}^{d i s s}
$$

Elastic cross sections are taken as

$$
\sigma_{c \bar{c} \tilde{B}}^{e l}=1.9 \mathrm{mb}, \quad \sigma_{c \bar{c} \tilde{m}}^{e l}=\frac{2}{3} \sigma_{c \bar{c} \tilde{B}}^{e l} .
$$

Furthermore, elastic interactions of a charm quark (antiquark) are modeled by the scattering of an unformed $D$ or $D^{*}$ meson on pre-hadrons with only light quarks as

$$
\sigma_{D \tilde{B}}^{e l}=3.9 \mathrm{mb}, \quad \sigma_{D \tilde{m}}^{e l}=\frac{2}{3} \sigma_{D \tilde{B}}^{e l} .
$$

In this way we may incorporate in HSD some dynamics of quark-antiquark pairs with a medium that has not yet formed the ordinary hadrons. However, it has to be stressed that further explicit partonic degrees of freedom, i.e. gluons and their mutual interactions as well as gluon interactions with quarks 


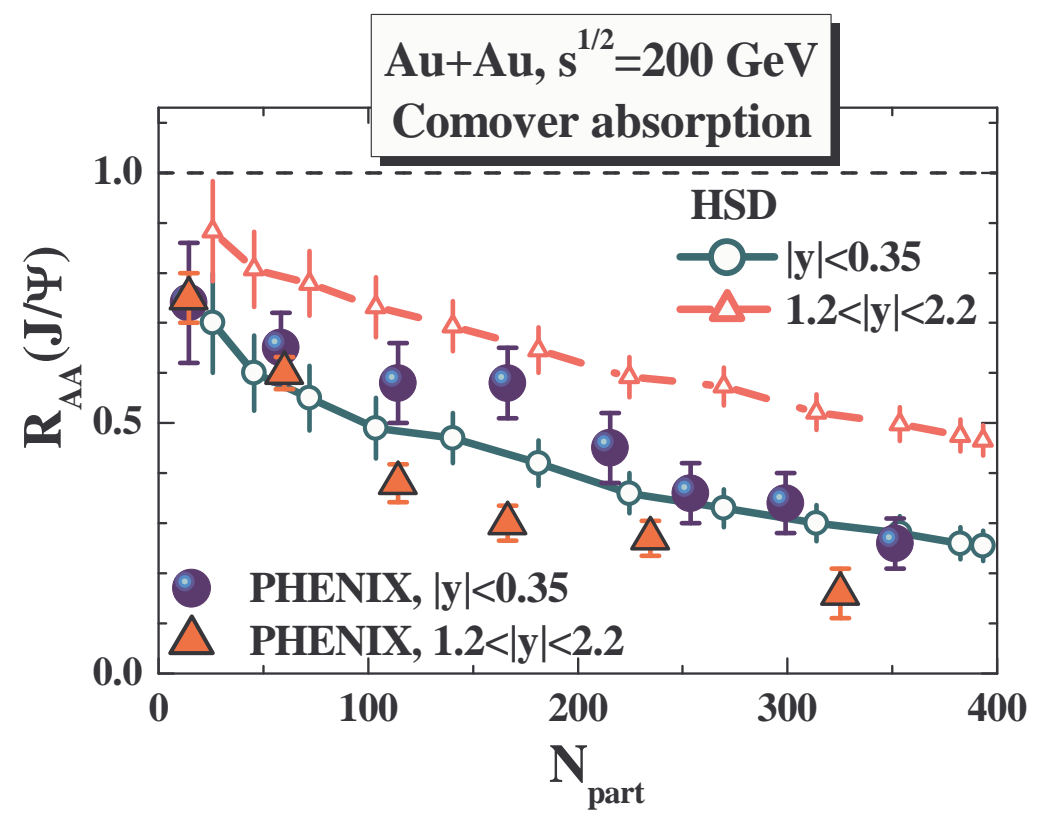

Fig. 7. The $J / \Psi$ nuclear modification factor $R_{A A}$ (18) for $\mathrm{Au}+\mathrm{Au}$ collisions at $\sqrt{s}=200 \mathrm{AGeV}$ as a function of the number of participants $N_{\text {part }}$ in comparison to the data from [41] for midrapidity (full circles) and forward rapidity (full triangles). The HSD results for the purely hadronic 'comover' scenario are displayed in terms of the lower (green solid) line with open circles for midrapidity $J / \Psi^{\prime} s(|y| \leq 0.35)$ and in terms of the upper (red dashed) line with open triangles for forward rapidity $(1.2 \leq|y| \leq 2.2)$.

and antiquarks, are not taken into account in the present HSD approach. Therefore, we do not expect to reproduce any details of the measured $J / \Psi$ yield. The study of this particular model situation is motivated first of all by the possibility to assess the conceptual influence of charm scattering on pre-hadrons (in the early reaction phase) on the final rapidity distribution of the $J / \Psi$ 's (see below).

\section{Comparison to data}

In the transport approach we calculate the $J / \Psi$ survival probability $S_{J / \Psi}$ and the nuclear modification factor $R_{A A}$ as

$$
S_{J / \Psi}=\frac{N_{\text {fin }}^{J / \Psi}}{N_{B B}^{J / \Psi}}, \quad R_{A A}=\frac{d N_{A A}^{J / \Psi} / d y}{N_{\text {coll }} \cdot d N_{p p}^{J / \Psi} / d y},
$$

where $N_{\text {fin }}^{J / \Psi}$ and $N_{B B}^{J / \Psi}$ denote the final number of $J / \Psi$ mesons and the number of $J / \Psi$ 's produced initially by $B B$ reactions, respectively. Note that $N_{\text {fin }}^{J / \Psi}$ 


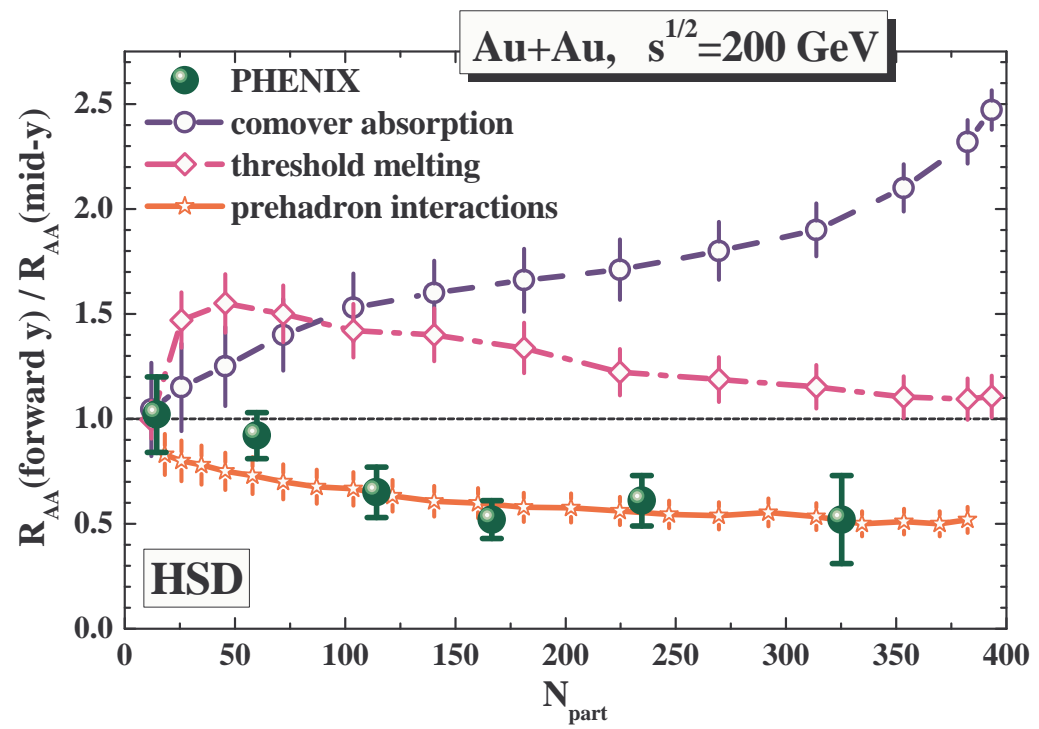

Fig. 8. The ratio of the nuclear modification factors $R_{A A}$ at mid-rapidity $(|y|<0.35)$ and at forward rapidity $(1.2<|y|<2.2)$ vs centrality in $A u+A u$ collisions at $\sqrt{s}=200 \mathrm{GeV}$. The HSD results in the purely hadronic scenario ('comover absorption') are displayed in terms of the blue dashed line (with open circles) and in case of the 'threshold melting' scenario in terms of the violet dot-dashed line (with open squares). The error bars on the theoretical results indicate the statistical uncertainty due to the finite number of Monte-Carlo events in the calculations. The lower full green dots represent the data of the PHENIX Collaboration [41. Note that the data have an additional systematic uncertainty of $\pm 14 \%$. The lower solid (red) line with stars gives the result for the 'comover absorption' scenario when including additional pre-hadronic interactions with charm (see text).

includes the decays from the final $\chi_{c}$. In (18),$d N_{A A}^{J / \Psi} / d y$ denotes the final yield of $J / \Psi$ in $A A$ collisions, $d N_{p p}^{J / \Psi} / d y$ is the yield in elementary $p p$ reactions while $N_{\text {coll }}$ is the number of initial binary collisions.

The suppression of charmonia by the 'comover' dissociation channels within the model described in [11] for a matrix element squared $\left|M_{0}\right|^{2}=0.18 \mathrm{fm}^{2} / \mathrm{GeV}^{2}$ has been presented already in Ref. [12] as well as the results for the 'threshold melting scenario' employing the thresholds $\varepsilon_{J / \Psi}=16 \mathrm{GeV} / \mathrm{fm}^{3}, \varepsilon_{\chi_{c}}=\varepsilon_{\Psi^{\prime}}=2$ $\mathrm{GeV} / \mathrm{fm}^{3}$. Note that the charmonium reformation channels by $D+\bar{D}$ channels had been incorporated, too ( $c f$. Ref. [14]). Since the PHENIX Collaboration has released a new data set we compare our calculations with the most recent PHENIX data [1] in Fig. [7 for the $J / \Psi$ nuclear modification factor $R_{A A}$ (18) for $\mathrm{Au}+\mathrm{Au}$ collisions at $\sqrt{s}=200 \mathrm{AGeV}$ as a function of the number of participants $N_{\text {part }}$ for midrapidity (full circles) and forward rapidity (full triangles). The HSD results for the purely hadronic 'comover' scenario are displayed in terms of the lower (blue solid) line with open circles for midrapidity $J / \Psi^{\prime} s$ $(|y| \leq 0.35)$ and in terms of the upper (red dashed) line with open triangles for forward rapidity $(1.2 \leq|y| \leq 2.2)$. The numerical results appear accept- 


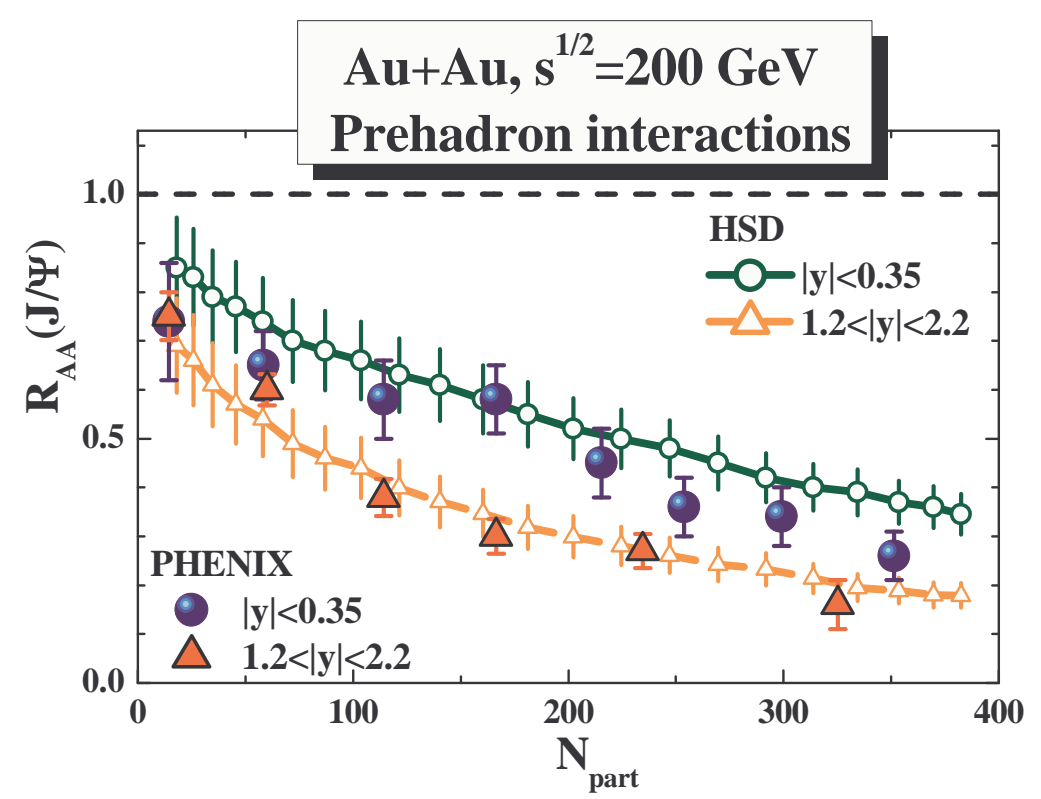

Fig. 9. The $J / \Psi$ nuclear modification factor $R_{A A}$ (18) for $\mathrm{Au}+\mathrm{Au}$ collisions at $\sqrt{s}=200 \mathrm{AGeV}$ as a function of the number of participants $N_{\text {part }}$ in comparison to the data from 41 for midrapidity (full circles) and forward rapidity (full triangles). The HSD results for the hadronic 'comover' scenario including additionally pre-hadronic interactions of charm according to (14) - (17) are displayed in terms of the upper (green solid) line with open circles for midrapidity $J / \Psi^{\prime} s(|y| \leq 0.35)$ and in terms of the lower (orange dashed) line with open triangles for forward rapidity $(1.2 \leq|y| \leq 2.2)$.

able at midrapidity $(|y| \leq 0.35)$ but the even larger suppression at forward rapidity (seen experimentally) is fully missed (cf. Ref. [12]).

The failure of the traditional 'comover absorption' model as well as 'threshold melting' scenario at the top RHIC energy is most clearly seen in the centrality dependence of the ratio of the nuclear modification factors $R_{A A}$ at forward rapidity $(1.2<|y|<2.2)$ and at mid-rapidity $(|y|<0.35)$ as shown in Fig. 8 . The HSD results in the purely hadronic scenario ('comover absorption') are displayed in terms of the blue dashed line (with open circles) and in case of the 'threshold melting' scenario in terms of the dot-dashed violet line (with open squares). The error bars on the theoretical results indicate the statistical uncertainty due to the finite number of Monte-Carlo events in the calculations. The lower full green dots in Fig. 8 represent the corresponding data of the PHENIX Collaboration [41] which show a fully different pattern as a function of centrality (here given in terms of the number of participants $N_{\text {part }}$ ). The failure of these 'standard' suppression models at RHIC has lead to the conclusion in Ref. [12] that the hadronic 'comover absorption and recombination' model is falsified by the PHENIX data and that strong interactions in the pre-hadronic (or partonic) phase should be necessary to explain the large suppression at forward rapidities. 

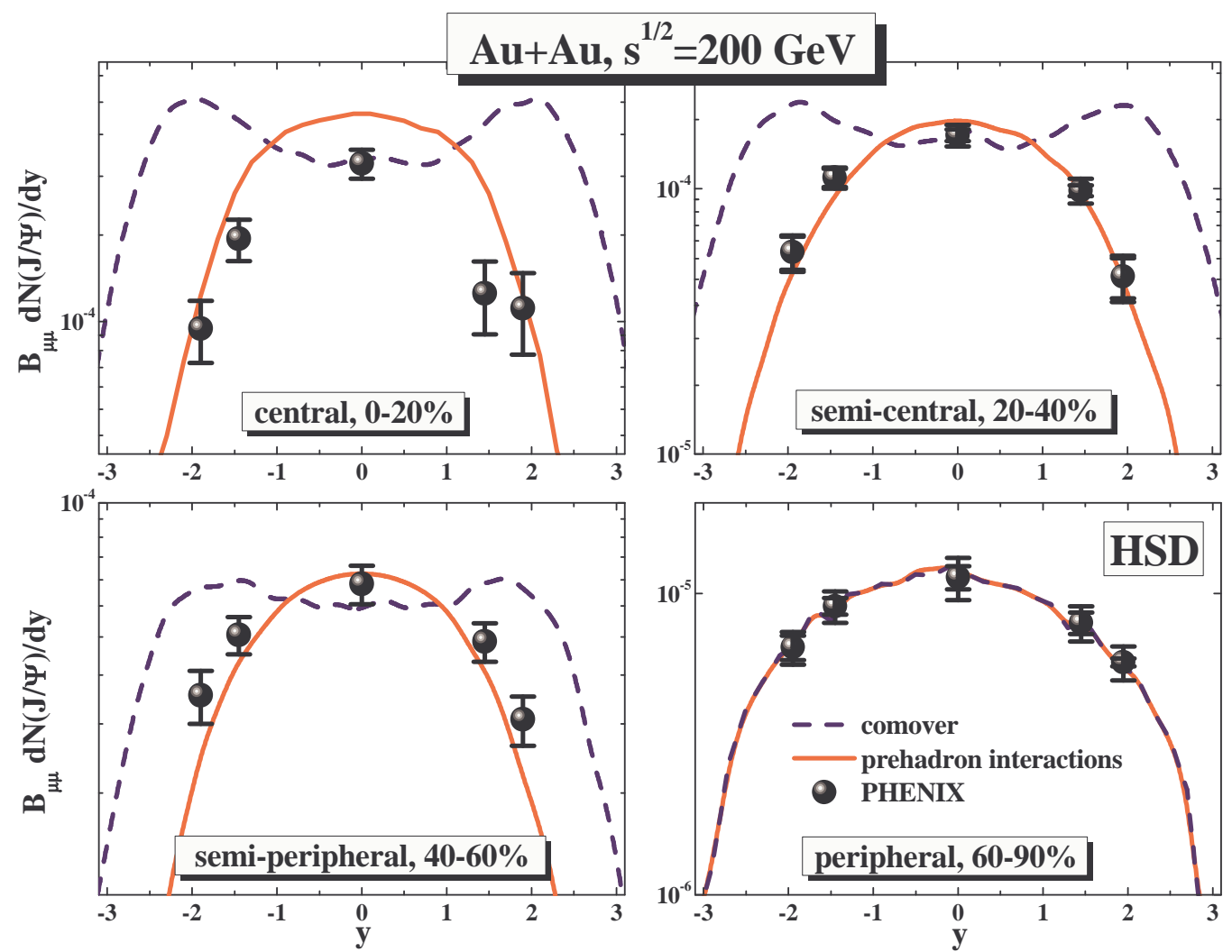

Fig. 10. The rapidity distribution $d N_{J / \Psi} / d y$ for different centralities from the standard 'comover' model (dashed blue lines) and the 'comover' model with additional pre-hadronic interactions of charm according to (14) - (17) (solid red lines). The full dots show the respective data from the PHENIX Collaboration [41. The calculated lines have been smoothed by a spline algorithm. The reactions are $\mathrm{Au}+\mathrm{Au}$ at $\sqrt{s}$ $=200 \mathrm{GeV}$.

In this work we follow up the latter idea and incorporate in the 'comover scenario' the additional pre-hadronic cross sections (14) - (17) for the early charm interactions to have a first glance at the dominant effects. The $J / \Psi$ suppression pattern in this case is shown in Fig. 9] in comparison to the same data as in Fig. 7. Now, indeed, the suppression pattern for central and forward rapidities becomes rather similar to the data within the statistical accuracy of the calculations. Indeed, the ratio of $R_{A A}$ at forward rapidity to midrapidity now follows closely the experimental trend as seen in Fig. 8 by the lower red solid line.

Some further information may be gained from the $J / \Psi$ rapidity distributions in $\mathrm{Au}+\mathrm{Au}$ collisions at RHIC. The latter distribution is shown in Fig. 10 in comparison to the PHENIX data for central collisions (upper l.h.s.), semicentral (upper r.h.s.), semi-peripheral (lower l.h.s.) and peripheral reactions (lower r.h.s.) for the standard 'comover' scenario (dashed blue lines) and the 'comover' model including additionally pre-hadronic interactions of charm according to (14) - (17) (solid red lines). Whereas for peripheral reactions these 
additional early interactions practically play no role, the latter lead to a narrowing of the $J / \Psi$ rapidity distribution with the centrality of the collision (roughly in line with the data). In the standard 'comover' model an opposite trend is seen: here the interactions of charmonia with formed hadrons produce a dip in the rapidity distribution at $y \approx 0$ which increases with centrality since the density of formed hadrons increases accordingly around midrapidity. Since the total number of produced $c \bar{c}$ pairs is the same (for the respective centrality class) and detailed balance is incorporated in the reaction rates we find an surplus of $J / \Psi$ at more forward rapidities. The net result is a broadening of the $J / \Psi$ rapidity distribution with centrality opposite to the trend observed in experiment.

Summarizing the results displayed in Figs. 7 - 10 we like to point out that the hadronic 'comover' dynamics for charmonium dissociation and recreation - as well as the standard charmonium 'melting' scenario - do not match the general dependences of the $J / \Psi$ in rapidity and centrality as seen by the PHENIX Collaboration. In fact, a narrowing of the $J / \Psi$ rapidity distribution cannot be achieved by comover interactions with formed hadrons since the latter appear too late in the collision dynamics. Only when including early prehadronic interactions with charm a dynamical narrowing of the charmonium rapidity distribution with centrality can be achieved as demonstrated more schematically within our pre-hadronic interaction model. Consequently, the PHENIX data on $J / \Psi$ suppression demonstrate the presence and important impact of pre-hadronic or partonic interactions in the early charm dynamics. This finding is line with earlier studies in Refs. [14,18,19] demonstrating the necessity of non-hadronic degrees of freedom in the early reaction phase for the elliptic flow $v_{2}$, the suppression of hadrons at high transverse momentum $p_{T}$ and far-side jet suppression in central $\mathrm{Au}+\mathrm{Au}$ collisions at $\mathrm{RHIC}$ energies.

\section{Predictions, excitation functions and comparison to the statisti- cal hadronization model}

In this Section we continue with predictions for future measurements as well as model comparisons in order to allow for an experimental discrimination between the model concepts.

\section{$7.1 \Psi^{\prime}$ as an independent probe}

As pointed in Ref. [11] an independent measurement of $\Psi^{\prime}$ will provide further information on the charm reaction dynamics and final charmonium formation. For instance, a leveling off of the $\Psi^{\prime}$ to $J / \Psi$ ratio with increasing centrality 


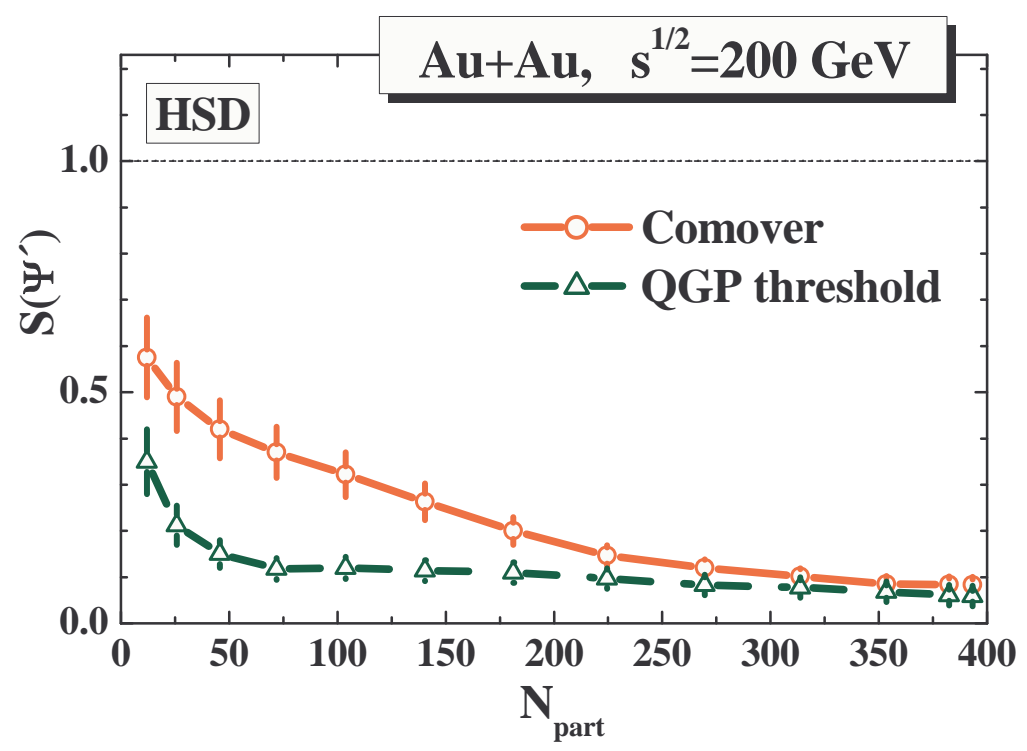

Fig. 11. Survival probability of $\Psi^{\prime}$ in $A u+A u$ reactions at $\sqrt{s}=200 \mathrm{GeV}$ in the 'threshold melting' (dashed line with triangles) and 'comover' suppression (solid line with circles) approaches, see text for details.

would be a signal for charm chemical equilibration in the medium [3,4,5]. Additionally, it provides a very clear distinction between the 'threshold melting' scenario and the 'comover' approach. Since detailed predictions for the $\Psi^{\prime}$ to $J / \Psi$ ratio as a function of centrality have already been presented in Ref. [1] for FAIR and SPS energies we here complement the latter studies by results for the top RHIC energy although the suppression of $\Psi^{\prime}$ mesons has not yet been measured at RHIC.

In Fig 11, we accordingly present the $\Psi^{\prime}$ survival probability $S_{\Psi^{\prime}}$ defined as

$$
S_{\Psi^{\prime}}=\frac{N_{f i n}^{\Psi^{\prime}}}{N_{B B}^{\Psi^{\prime}}},
$$

for $A u+A u$ at $\sqrt{s}=200 \mathrm{GeV}$. In equation (19), $N_{f i n}^{\Psi^{\prime}}$ and $N_{B B}^{\Psi^{\prime}}$ denote the number of final $\Psi^{\prime}$ mesons and of those produced initially by $B B$ reactions, respectively. One can see from Fig 11 that the 'threshold melting' scenario at RHIC predicts an almost complete melting of $\Psi^{\prime}$, while a hadronic 'comover' absorption scenario shows a gradual decrease of the number of $\Psi^{\prime}$ with $N_{\text {part }}$. Similar differences between the models have also been found at SPS energies [11] where the presently available data sets clearly favor the 'comover' model. On the other hand our predictions for the top RHIC energy imply that the $\Psi^{\prime}$ signal will be very low for mid-central and central $\mathrm{Au}+\mathrm{Au}$ collisions such that actual measurements will turn out to be very demanding. 

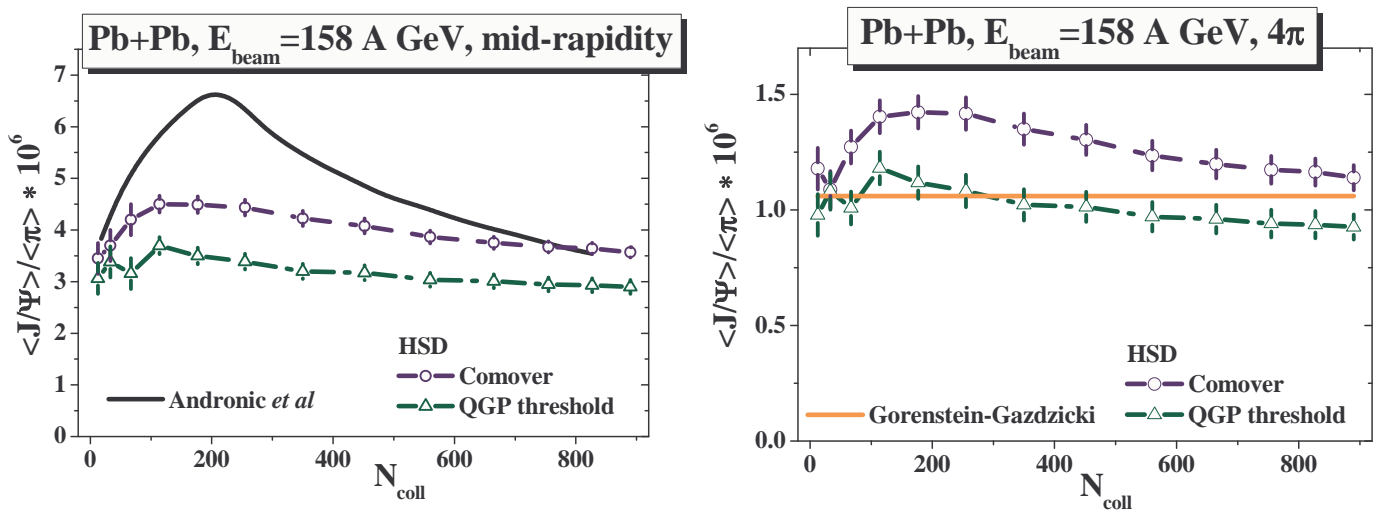

Fig. 12. Ratio of the averaged $J / \Psi$ to $\pi$ multiplicity for $P b+P b$ at the SPS beam energy of $158 \mathrm{~A} \cdot \mathrm{GeV}$ at mid-rapidity (l.h.s.) and in full $4 \pi$ acceptance (r.h.s.) as a function of the number of binary collisions $N_{\text {coll }}$ for the different suppression scenarios implemented in HSD - the 'comover' model (dashed blue line with open circles) and the 'threshold melting' scenario (green dot-dashed line with open triangles) - in comparison to the statistical model by Gorenstein and Gazdzicki [2] (r.h.s.; straight orange line) and the statistical hadronization model by Andronic et al. [3] (1.h.s.; solid black line).
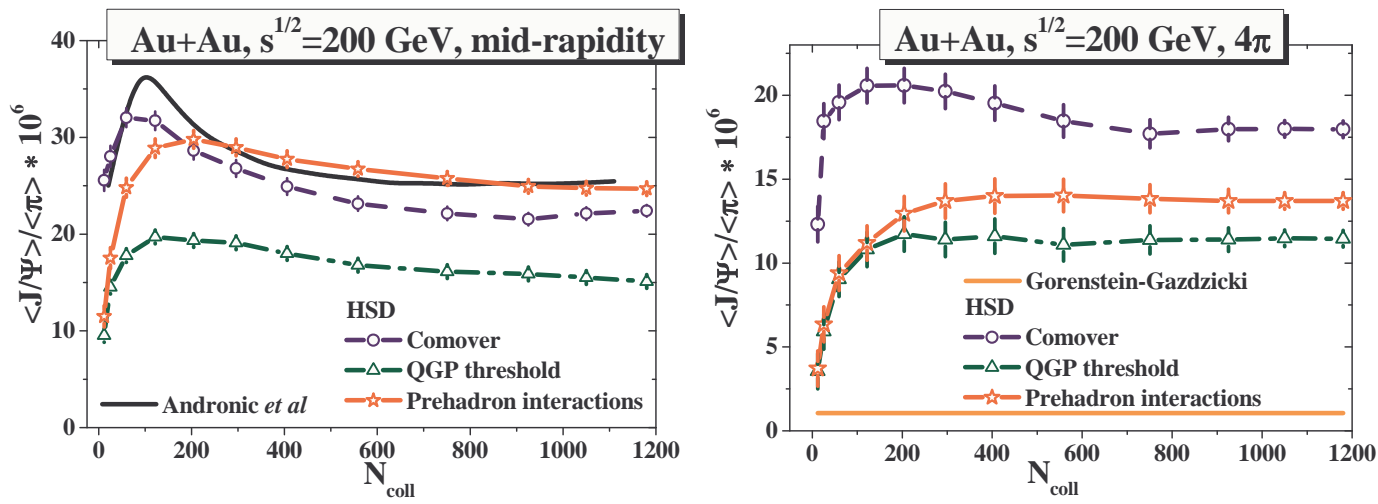

Fig. 13. Same as Fig. 12 but for $A u+A u$ at the top RHIC energy of $\sqrt{s}=200 \mathrm{GeV}$. The red solid line shows additionally the result of the 'comover' model including the pre-hadronic charm interactions (see text).

\subsection{Testing the assumption of statistical hadronization}

The assumption of statistical hadronization - i.e. of $J / \Psi$ 's being dominantly produced at hadronization in a purely statistically fashion according to available phase space and the number of available $c$ and $\bar{c}$ quarks - leads to a scaling of the $\langle J / \Psi\rangle /\langle h\rangle$ ratio with the system size [2], where $\langle h\rangle$ is the average hadron multiplicity. Since $\langle h\rangle \sim\langle\pi\rangle$, we calculate the ratio $\langle J / \Psi\rangle /\langle\pi\rangle$ in HSD in the different scenarios for charmonium suppression:

- 'threshold melting' + recombination via $D \bar{D} \rightarrow c \bar{c}+m$ including the backward reactions $c \bar{c}+m \rightarrow D \bar{D}$, 
- hadronic ('comover') absorption: $D \bar{D} \rightarrow c \bar{c}+m$ and the backward reactions $c \bar{c}+m \rightarrow D \bar{D}$

- 'prehadron interactions': $D \bar{D} \rightarrow c \bar{c}+m$ and the backward reactions $c \bar{c}+m \rightarrow$ $D \bar{D}$ as well as early pre-hadronic charm interactions as described in Section 6 .

The results of our calculations are shown in Fig. 12 together with the prediction of the statistical model of Gorenstein and Gazdzicki [2] for the full phase space (straight orange line; r.h.s.) and the statistical hadronization model by Andronic et al. [3,79] for mid-rapidity (solid black line; l.h.s.) for $\mathrm{Pb}+\mathrm{Pb}$ at $158 \mathrm{~A} \cdot \mathrm{GeV}$. The centrality dependence here is given by the number of initial binary collisions $N_{\text {coll }}$. The actual comparison in Fig. 13 indicates that the statistical model by Andronic et al. [3] predicts a sizeably larger $J / \Psi$ to $\pi$ ratio at midrapidity for peripheral and semi-peripheral reactions than the microscopic HSD results for the different scenarios. For central reactions - where an approximate equilibrium is achieved - all scenarios give roughly the same ratio. In full $4 \pi$ phase space the HSD results indicate also a slightly higher $J / \Psi$ to $\pi$ ratio in the 'comover' model relative to the 'melting' scenario but both ratios only weakly depend on centrality roughly in line with the statistical model of Gorenstein and Gazdzicki [2] (orange straight line). Consequently, only peripheral reactions of heavy nuclei might be used to disentangle the different scenarios at top SPS energies at midrapidity (or in full phase space).

The situation is different for $\mathrm{Au}+\mathrm{Au}$ collisions at the top RHIC energy as may be extracted from Fig. 13 where the $J / \Psi$ to pion ratio (1.h.s.: at midrapidity; r.h.s.: for $4 \pi$ acceptance) is shown again as a function of $N_{\text {coll }}$. The standard 'comover' model (dashed blue lines) is only shown for reference but is unrealistic according to the analysis in Section 6. We find that the 'comover' model with early pre-hadronic charm interactions (solid red line with stars, l.h.s.) is very close to the statistical hadronization model [3] (solid black line) at midrapidity except for very peripheral collisions. The 'threshold melting' scenario follows the trend in centrality but is down by about $30 \%$. Thus at midrapidity there is no essential extra potential in differentiating the scenarios. Considering the full $4 \pi$ acceptance (r.h.s.) we find a practically constant $J / \Psi$ to pion ratio for $N_{\text {coll }}>200$ from the HSD calculations as expected from the statistical model, however, the early model of Gorenstein and Gazdzicki [2] is down by about a factor of $\sim 10$ (and may be ruled out by present data).

\subsection{Excitation functions}

In this Subsection we present the excitation functions for the $J / \Psi$ survival probability in $\mathrm{Au}+\mathrm{Au}$ collisions from FAIR to top RHIC energies in the different scenarios in order to allow for a further distinction between the different 

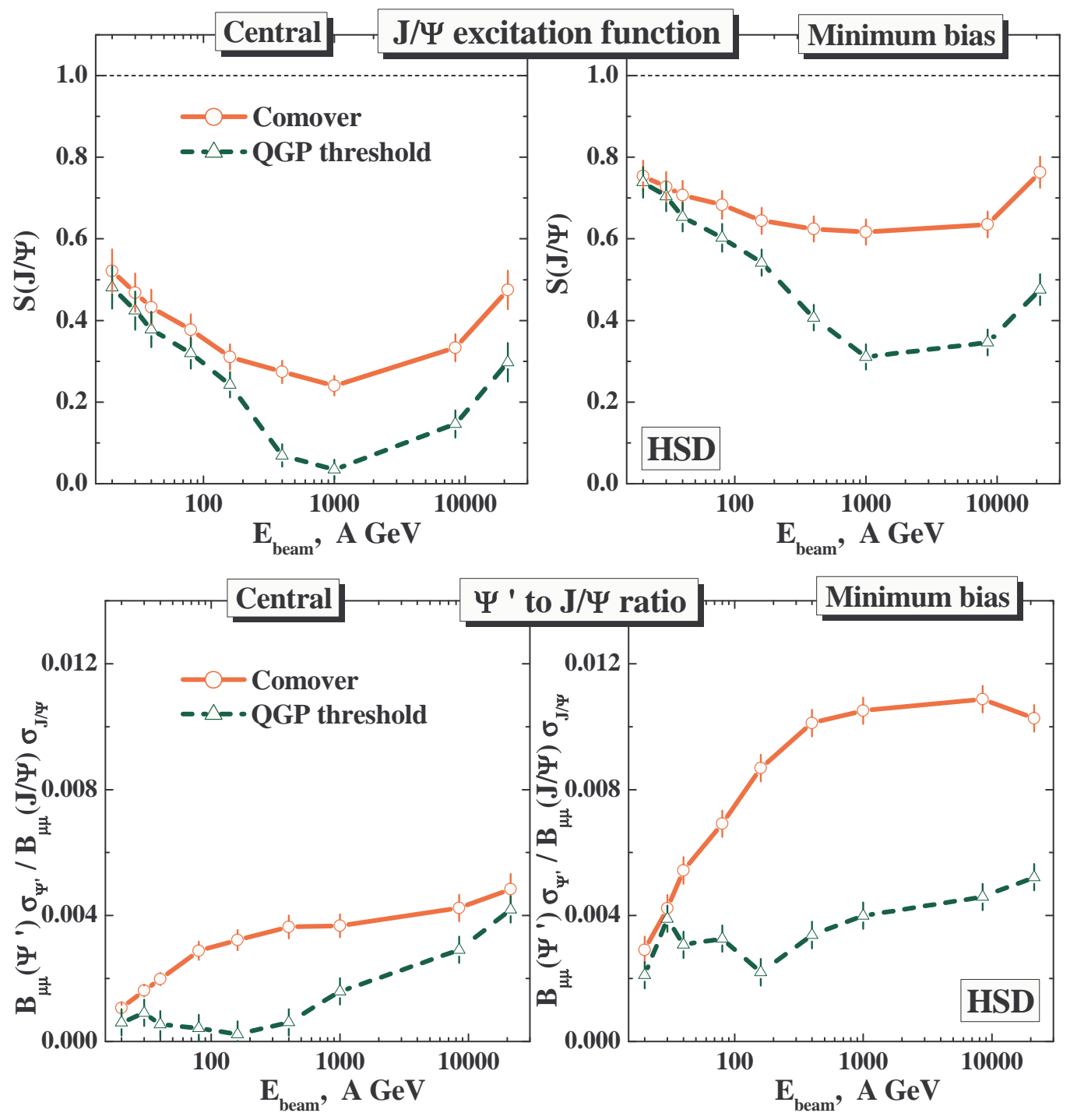

Fig. 14. upper part: The excitation function for the $J / \Psi$ survival probability in the 'QGP threshold melting + hadronic recombination' scenario (dashed green lines with triangles) and the 'comover absorption + recombination' model (solid red lines with circles) for central (l.h.s.) and minimum bias $\mathrm{Au}+\mathrm{Au}$ reactions (r.h.s.) as a function of the beam energy. Lower part: The $\Psi^{\prime}$ to $J / \Psi$ ratio for the same reactions as in the upper part of the figure in the 'QGP threshold melting + hadronic recombination' scenario (dashed green lines with triangles) and the 'comover absorption + recombination' model (upper solid red lines with circles ).

concepts. The results of our HSD calculations are presented in the upper part of Fig. 14 for the 'QGP threshold melting + hadronic recombination' scenario (dashed green lines with open triangles) and the 'comover absorption + recombination' model (solid red lines with open circles) for central (l.h.s.) and minimum bias (r.h.s.) Au+Au reactions as a function of the beam energy. We find that from FAIR energies of 20 - $40 \mathrm{~A} \cdot \mathrm{GeV}$ up to top SPS energies of $158 \mathrm{~A}$. $\mathrm{GeV}$ there is no significant difference for the $J / \Psi$ survival probability in case of 


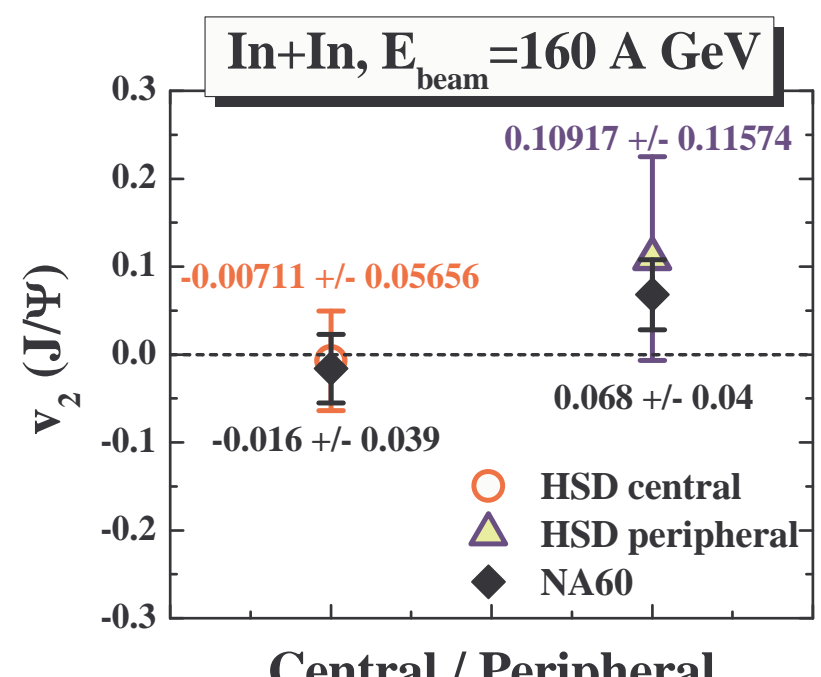

Fig. 15. Elliptic flow $v_{2}$ of $J / \Psi$ 's produced in central and peripheral $I n+\operatorname{In}$ collisions at $158 \mathrm{~A} \cdot \mathrm{GeV}$ beam energy in the hadronic 'comover' mode of HSD (open circle and open triangle) compared to the NA60 data [80] represented by black diamonds.

central collisions. The differences here show mainly up in the full RHIC energy range where the 'QGP threshold melting + hadronic recombination' scenario leads to a substantially lower $J / \Psi$ survival probabilities. In case of minimum bias collisions the 'comover absorption + recombination' model (solid lines) leads to a roughly energy independent $J / \Psi$ survival probability whereas the 'QGP threshold melting + hadronic recombination' scenario shows lower $J / \Psi$ survival probabilities (lower dashed green lines) for laboratory energies above $\sim 100 \mathrm{~A} \cdot \mathrm{GeV}$ due to a larger initial melting of $J / \Psi$ at high energy density.

A clearer distinction between the different concepts is offered by the excitation functions for the $\Psi^{\prime}$ to $J / \Psi$ ratio in $\mathrm{Au}+\mathrm{Au}$ collisions. The calculated results are shown in the lower part of Fig. 14 for the 'QGP threshold melting + hadronic recombination' scenario (dashed green lines with open triangles) and the 'comover absorption + recombination' model (solid red lines) for central (l.h.s.) and minimum bias reactions (r.h.s.). Here the $\Psi^{\prime}$ is already melting away in central $\mathrm{Au}+\mathrm{Au}$ reactions in the 'QGP threshold melting' scenario at bombarding energies above $40 \mathrm{~A} \cdot \mathrm{GeV}$ whereas a substantial amount of $\Psi^{\prime}$ survives in the 'comover absorption + recombination' model. Thus measurements of $\Psi^{\prime}$ suppression at the lower SPS or top FAIR energies will clearly distinguish between the different model concepts. 


\subsection{Elliptic flow of charm}

The elliptic flow of particles defined as

$$
v_{2}\left(y, p_{T}\right)=\left\langle\frac{p_{x}^{2}-p_{y}^{2}}{p_{T}^{2}}\right\rangle_{y, p_{T}}
$$

(with $p_{T}^{2}=p_{x}^{2}+p_{y}^{2}$ ) provides additional information on the collective currents and pressure evolution in the early phase of the complex reaction [81] since it is driven by different pressure gradients in case of nonvanishing spatial anisotropy $\epsilon_{2}=<\frac{y^{2}-x^{2}}{y^{2}+x^{2}}>$. Since $\epsilon_{2}$ decreases fast during the expansion of a noncentral reaction the magnitude of $v_{2}$ gives information about the interaction strength or interaction rate of the early medium.

In Fig. 15 we test the HSD result for $v_{2}(J / \Psi)$ at SPS in the purely hadronic 'comover' scenario in comparison to the data for $v_{2}$ of the NA60 collaboration for In+In collisions [80]. In central collisions the elliptic flow is practically zero both in the calculation as well as in the experiment whereas in peripheral reactions a nonzero flow emerges. The agreement (within error bars) between the theory and the data indicates that in line with the reproduction of the $J / \Psi$ suppression data [11] the low amount of $v_{2}$ does not point towards additional strong partonic interactions. Consequently, the present measurements of $J / \Psi$ elliptic flow at SPS energies do not provide further constraints on the model assumptions.

The situation, however, is different for the collective flow of $D$-mesons at top RHIC energies. In Fig. 16 we show the elliptic flow of $D$-mesons produced in $A u+A u$ collisions at $\sqrt{s}=200 \mathrm{GeV}$ as a function of the transverse momentum $p_{T}$ in HSD (solid blue line with open circles) compared to the PHENIX data [82] on $v_{2}$ of non-photonic electrons. Here the elliptic flow of $D$-mesons is clearly underestimated in the standard HSD model (cf. Ref. [35]). Only when including pre-hadronic charm interactions - as described in Section 6 - the elliptic flow increases (red line with open stars) but still stays clearly below the PHENIX data for $p_{T}<2 \mathrm{GeV} / \mathrm{c}$. We thus have to conclude that the modeling of charm interactions by pre-hadronic interactions - as described in Section 6 does not provide enough interaction strength in the early phase of the collision. Quite remarkably this finding is again fully in line with the underestimation of high $p_{T}$ hadron suppression [18] as well as far-side jet suppression [19] in the pre-hadronic interaction model. Independently, also the charm collective flow points towards strong partonic interactions in the early reaction phase beyond the pre-hadronic scattering incorporated so far.

Since a large fraction of $J / \Psi$ 's in central $\mathrm{Au}+\mathrm{Au}$ collisions at RHIC are created by $D-\bar{D}$ recombination, the elliptic flow of $J / \Psi$ 's obtained from HSD in the 


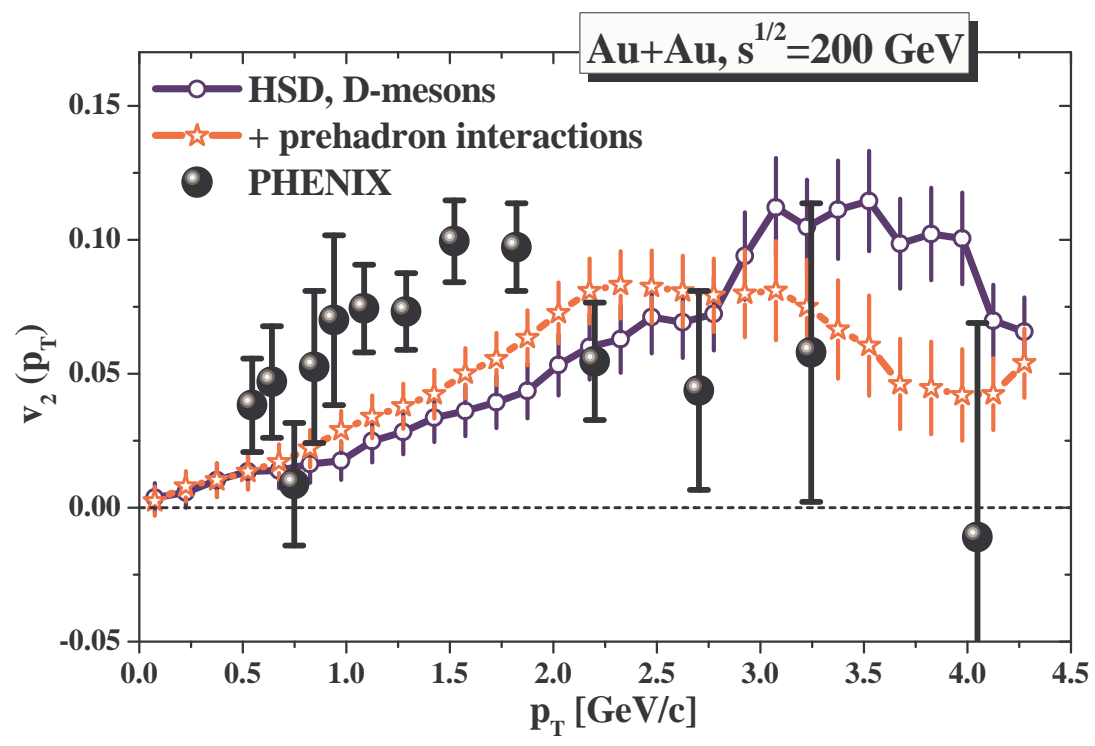

Fig. 16. Elliptic flow of $D$-mesons produced in $A u+A u$ collisions at $\sqrt{s}=200 \mathrm{GeV}$ as a function of $p_{T}$ from HSD (solid blue line with open circles) in comparison to the PHENIX data 82] on $v_{2}$ of non-photonic electrons. The red line with open stars shows the HSD result for the $v_{2}$ of $D$-mesons when including additionally pre-hadronic charm interactions as described in Section 6 .

comover (purely hadronic) case is comparatively small, too, and should not be in accord with future experimental data. We consequently discard an explicit representation of the $J / \Psi$ elliptic flow at RHIC energies since the calculations show the $v_{2}$ of charmonium to be very close to the $D$-meson flow within error bars.

\section{Summary}

Our present study essentially completes the investigations of charm production, propagation and chemical reactions within the HSD transport approach initiated more than a decade ago [30,31]. The present systematic investigation extends earlier work to RHIC energies and clearly shows - as advocated before [12] - that the traditional concepts of 'charmonium melting' in a QGP state as well as the hadronic 'comover absorption and recreation model' are in severe conflict with the data from the PHENIX Collaboration at RHIC energies whereas both model assumptions work reasonably well at top SPS energies [11].

The essential new result of this work is that (at top RHIC energies) we find evidence for strong interactions of charm with the pre-hadronic medium from comparison to recent data from the PHENIX Collaboration 41. In particular, pre-hadronic interactions (of unformed hadrons) with charm lead to 
dramatically different rapidity distributions for $J / \Psi$ 's and consequently to a substantially modified ratio $R_{A A}^{\text {forward }}(J / \Psi)$ to $R_{A A}^{\text {mid }}(J / \Psi)$ compared to earlier calculations/predictions.

Further results of the present microscopic transport study may be stated as follows:

- The $J / \Psi$ suppression in $d+A u$ collisions at $\sqrt{s}=200 \mathrm{GeV}$ is only roughly compatible with the charmonium absorption on nuclei as observed at SPS energies in $p+A$ reactions. We find a clear indication for shadowing effects at forward rapidity, but a conclusive answer about the size of this effect is not possible due to the statistical error bars in both the experimental data and the calculations. A proper answer can only be given by future high statistics data that allow to fix the scale of shadowing in a model independent way.

- The $\Psi^{\prime}$ to $J / \Psi$ ratio is found to be crucial in disentangling the different charmonium absorption scenarios. This result essentially emerges from the early dissociation of $\Psi^{\prime}$ above the critical energy density $\epsilon_{c} \approx 1 \mathrm{GeV} / \mathrm{fm}^{3}$ in the 'QGP melting scenario' whereas the $\Psi^{\prime}$ in the 'comover model' survives to higher energy densities.

- A comparison of the transport calculations to the statistical model of Gorenstein and Gazdzicki [2] (in $4 \pi$ acceptance) or the statistical hadronization model of Andronic et al. [3] (at midrapidity) shows differences in the energy as well as centrality dependence of the $J / \Psi$ to pion ratio, which might be exploited experimentally to discriminate the different concepts.

- The collective flow of charm in the HSD transport appears compatible with the data at SPS energies, but the data are substantially underestimated at top RHIC energies (cf. Fig. 16). This not only holds for the standard hadronic comover scenario, but also when including interactions of charm with pre-hadronic states (unformed hadrons). Consequently the large elliptic flow $v_{2}$ of charm seen experimentally has to be attributed to early interactions of non-hadronic degrees of freedom.

The open problem - and future challenge - is to incorporate explicit partonic degrees of freedom in the description of relativistic nucleus-nucleus collisions and their transition to hadronic states in a microscopic transport approach. On the experimental side, further differential spectra of charmonia and open charm mesons then will constrain the transport properties of charm in the early non-hadronic phase of nucleus-nucleus collisions at RHIC (and possibly at SPS or even FAIR energies). 


\section{Acknowledgements}

We acknowledge stimulating correspondence with A. Andronic, T. Gunji, D. Kim and J. Skullerud as well as helpful discussions with P. Braun-Munzinger, M. Gorenstein, R. Granier de Cassagnac, K. Redlich, J. Stachel and H. Stöcker. Furthermore, O. L. and E.L.B. would like to thank the BMBF for financial support.

\section{References}

[1] P. Braun-Munzinger, D. Miskowiec, A. Drees, and C. Lourenco, Eur. Phys. J. C1, 123 (1998).

[2] M. Gazdzicki and M. I. Gorenstein, Phys. Rev. Lett. 83, 4009 (1999).

[3] A. Andronic, P. Braun-Munzinger, K. Redlich, and J. Stachel, Phys. Lett. B652, 259 (2007).

[4] P. Braun-Munzinger and J. Stachel, Phys. Lett. B490, 196 (2000).

[5] P. Braun-Munzinger and J. Stachel, Nucl. Phys. A690, 119 (2001).

[6] R. L. Thews, M. Schroedter, and J. Rafelski, Phys. Rev. C63, 054905 (2001).

[7] C. Lourenco and H. Wohri, Phys. Rept. 433, 127 (2006).

[8] B. Brambilla et al., CERN Yellow Report, CERN-2005-005 (2005), hepph/0412158.

[9] W. Cassing and E. L. Bratkovskaya, Phys. Rep. 308, 65 (1999).

[10] H. Weber, E. Bratkovskaya, W. Cassing, and H. Stöcker, Phys. Rev. C67, 014904 (2003).

[11] O. Linnyk, E. L. Bratkovskaya, W. Cassing, and H. Stöcker, Nucl. Phys. A786, 183 (2007).

[12] O. Linnyk, E. L. Bratkovskaya, W. Cassing, and H. Stöcker, Phys. Rev. C76, 041901 (2007).

[13] W. Cassing, E. L. Bratkovskaya, and A. Sibirtsev, Nucl. Phys. A691, 753 (2001).

[14] E. L. Bratkovskaya, W. Cassing, and H. Stöcker, Phys. Rev. C67, 054905 (2003).

[15] E. L. Bratkovskaya, A. P. Kostyuk, W. Cassing, and H. Stöcker, Phys. Rev. C69, 054903 (2004). 
[16] T. Falter, K. Gallmeister, W. Cassing, and U. Mosel, Phys. Rev. C70, 054609 (2004).

[17] T. Falter, K. Gallmeister, W. Cassing, and U. Mosel, Phys. Lett. B594, 61 (2004).

[18] W. Cassing, K. Gallmeister, and C. Greiner, Nucl. Phys. A735, 277 (2004).

[19] K. Gallmeister and W. Cassing, Nucl. Phys. A748, 241 (2005).

[20] A. Andronic, P. Braun-Munzinger, K. Redlich, and J. Stachel, Nucl. Phys. A789, 334 (2007).

[21] PHENIX, S. S. Adler et al., Phys. Rev. C71, 034908 (2005).

[22] The open HSD source code is available from the URL http://www.th.physik.uni-frankfurt.de/ 'brat/hsd.html.

[23] PHENIX, A. Adare et al., Phys. Rev. Lett. 98, 232002 (2007).

[24] W. Cassing, E. L. Bratkovskaya, and S. Juchem, Nucl. Phys. A674, 249 (2000).

[25] E705, L. Antoniazzi et al., Phys. Rev. Lett. 70, 383 (1993).

[26] WA11, Y. Lemoigne et al., Phys. Lett. B113, 509 (1982).

[27] NA50, B. Alessandro et al., Phys. Lett. B553, 167 (2003).

[28] K. Hagiwara et al., Phys. Rev. D66, 010001 (2002), (Review of Particle Properties).

[29] J. Geiss, C. Greiner, E. L. Bratkovskaya, W. Cassing, and U. Mosel, Phys. Lett. B447, 31 (1999).

[30] W. Cassing and E. L. Bratkovskaya, Nucl. Phys. A623, 570 (1997).

[31] W. Cassing and C. M. Ko, Phys. Lett. B396, 39 (1997).

[32] J. Geiss, W. Cassing, and C. Greiner, Nucl. Phys. A644, 107 (1998).

[33] R. Vogt, Phys. Rep. 310, 197 (1999).

[34] E672/E706, V. Abramov et al., FERMILAB-Pub-91/62-E, IFVE-91-9, Mar. 1991.

[35] E. L. Bratkovskaya, W. Cassing, H. Stöcker, and N. Xu, Phys. Rev. C71, 044901 (2005).

[36] STAR, A. Tai et al., J. Phys. G30, S809 (2004).

[37] Z. Lin and C. M. Ko, Phys. Rev. C62, 034903 (2000).

[38] Z. Lin and C. M. Ko, J. Phys. G27, 617 (2001).

[39] PHENIX, A. Adare et al., Phys. Rev. C77, 024912 (2008).

[40] NA60, A. Foerster et al., J. Phys. G32, S51 (2006). 
[41] PHENIX, A. Adare et al., (2006), nucl-ex/0611020.

[42] D. Kharzeev and R. L. Thews, Phys. Rev. C60, 041901 (1999).

[43] NA50, B. Alessandro et al., nucl-ex/0612012.

[44] NA50, G. Borges et al., J. Phys. G32, S381 (2006).

[45] K. Martins, D. Blaschke, and E. Quack, Phys. Rev. C51, 2723 (1995).

[46] C. Gerschel and J. Hufner, Z. Phys. C56, 171 (1992).

[47] PHENIX, S. S. Adler et al., Phys. Rev. Lett. 96, 012304 (2006).

[48] I. C. Arsene, L. Bravina, A. B. Kaidalov, K. Tywoniuk, and E. Zabrodin, (2007), arXiv:0708.3801 [hep-ph].

[49] A. Capella and E. G. Ferreiro, (2006), hep-ph/0610313.

[50] R. Vogt, Phys. Rev. C71, 054902 (2005).

[51] B. Kopeliovich, A. Tarasov, and J. Hufner, Nucl. Phys. A696, 669 (2001).

[52] A. Capella et al., (2007), arXiv:0712.4331 [hep-ph].

[53] PHENIX, R. Granier de Cassagnac, J. Phys. G34, S 955 (2007).

[54] B. Müller, Nucl. Phys. A661, 272c (1999).

[55] P. Braun-Munzinger and K. Redlich, Eur. Phys. J. C16, 519 (2000).

[56] K. Martins, D. Blaschke, and E. Quack, Phys. Rev. C51, 2723 (1995).

[57] C. Y. Wong, E. S. Swanson, and T. Barnes, Phys. Rev. C62, 045201 (2000).

[58] C. M. Ko, B. Zhang, X. N. Wang, and X. F. Zhang, Phys. Lett. B444, 237 (1998).

[59] F. O. Duraes, H. Kim, S. H. Lee, F. S. Navarra, and M. Nielsen, Phys. Rev. C68, 035208 (2003).

[60] W. Cassing, L. A. Kondratyuk, G. I. Lykasov, and M. V. Rzjanin, Phys. Lett. B513, 1 (2001).

[61] N. Armesto and A. Capella, Phys. Lett. B430, 23 (1998).

[62] P. Braun-Munzinger and K. Redlich, Nucl. Phys. A661, 546 (1999).

[63] L. Grandchamp and R. Rapp, Phys. Lett. B523, 60 (2001).

[64] L. Grandchamp and R. Rapp, Nucl. Phys. A709, 415 (2002).

[65] Z.-w. Lin and C. M. Ko, J. Phys. G27, 617 (2001).

[66] Z.-w. Lin and C. M. Ko, Phys. Rev. C65, 034904 (2002).

[67] T. Matsui and H. Satz, Phys. Lett. B178, 416 (1986). 
[68] H. Satz, Rep. Progr. Phys. 63, 1511 (2000).

[69] H. Satz, J. Phys. G32, R25 (2006).

[70] F. Karsch, D. Kharzeev, and H. Satz, Phys. Lett. B637, 75 (2006).

[71] G. Aarts, C. Allton, M. B. Oktay, M. Peardon, and J.-I. Skullerud, (2007), arXiv:0705.2198[hep-lat].

[72] S. Datta, F. Karsch, P. Petreczky, and I. Wetzorke, Phys. Rev. D69, 094507 (2004).

[73] P. Petreczky and K. Petrov, Phys. Rev. D70, 054503 (2004).

[74] RBC-Bielefeld, K. Petrov, PoS LAT2006, 144 (2006).

[75] O. Kaczmarek, PoS CPOD07, 043 (2007).

[76] A. Mocsy and P. Petreczky, (2007), arXiv:0706.2183 [hep-ph].

[77] H. van Hees and R. Rapp, Phys. Rev. C71, 034907 (2005).

[78] K. Gallmeister and T. Falter, Phys. Lett. B630, 40 (2005).

[79] A. Andronic, private communication.

[80] NA60, R. Arnaldi et al., Nucl. Phys. A783, 261 (2007).

[81] H. Stöcker, Nucl. Phys. A750, 121 (2005).

[82] PHENIX, A. Adare et al., Phys. Rev. Lett. 98, 172301 (2007). 